\title{
Region of Interest-Based Tamper Detection and Lossless Recovery Watermarking Scheme (ROI-DR) on Ultrasound Medical Images
}

\author{
Hui Liang Khor ${ }^{1}$ - Siau-Chuin Liew ${ }^{1}$ Jasni Mohd. Zain ${ }^{1}$
}

Published online: 3 January 2017

(C) Society for Imaging Informatics in Medicine 2016

\begin{abstract}
Tampering on medical image will lead to wrong diagnosis and treatment, which is life-threatening; therefore, digital watermarking on medical image was introduced to protect medical image from tampering. Medical images are divided into region of interest (ROI) and region of noninterest (RONI). ROI is an area that has a significant impact on diagnosis, whereas RONI has less or no significance in diagnosis. This paper has proposed ROI-based tamper detection and recovery watermarking scheme (ROI-DR) that embeds ROI bit information into RONI least significant bits, which will be extracted later for authentication and recovery process. The experiment result has shown that the ROI-DR has achieved a good result in imperceptibility with peak signal-to-noise ratio (PSNR) values approximately $48 \mathrm{~dB}$, it is robust against various kinds of tampering, and the tampered ROI was able to recover to its original form. Lastly, a comparative table with the previous research (TALLOR and TALLOR-RS watermarking schemes) has been derived, where these three watermarking schemes were tested under the same testing conditions and environment. The experiment result has shown that ROI-DR has achieved speed-up factors
\end{abstract}

Hui Liang Khor

hlkhorr@yahoo.com

Siau-Chuin Liew

liewsc@ump.edu.my

Jasni Mohd. Zain

jasni@ump.edu.my

1 Faculty of Computer Systems and Software Engineering, Universiti Malaysia Pahang, Lebuhraya Tun Razak, 26300 Gambang,

Kuantan, Pahang Darul Makmur, Malaysia of 22.55 and 26.65 in relative to TALLOR and TALLOR-RS watermarking schemes, respectively.

Keywords Watermarking $\cdot$ Region of interest (ROI) $\cdot$ Least significant bit (LSB) · Speed up · Tamper detection · Recovery

\section{Introduction}

Digital watermarking in medical images serves as a layer of protection against tampering. In medical image watermarking, a region of interest (ROI) is defined but untouched and the generated watermark is embedded in the region of non-interest (RONI). It is because ROI is the significant area for clinical diagnosis and modification was restricted to prevent any misdiagnosis from occurring. Several studies had divided a medical image into protection zone (ROI) and insertion zone (RONI) in their proposed watermarking schemes [1-3]. All these watermarking schemes focused on the intrinsic algorithm; therefore, it was usually conducted on a single frame medical image, but most of the medical images are formed by multiframes, such as ultrasound and MRI medical images. Wenbo D. et al. had introduced an improved version of duallayer watermarking scheme and exploited the 3-D property of volumetric (multiframes) Digital Imaging and Communications in Medicine (DICOM) images [4, 5]. They had utilized the advantage of 3-D property and manipulated them into their watermarking scheme algorithm. It is easy to migrate watermarking scheme from single frame to multiframe environment by using a control loop, such as loop to perform watermarking process on medical images sequentially, but it may be time-consuming; for example, the average processing time of Tamper Localization and Lossless Recovery (TALLOR) watermarking scheme was reported as $20.13 \mathrm{~s}$ per frame, and it would be $20.13 \mathrm{~min}$ for 60 frames of 


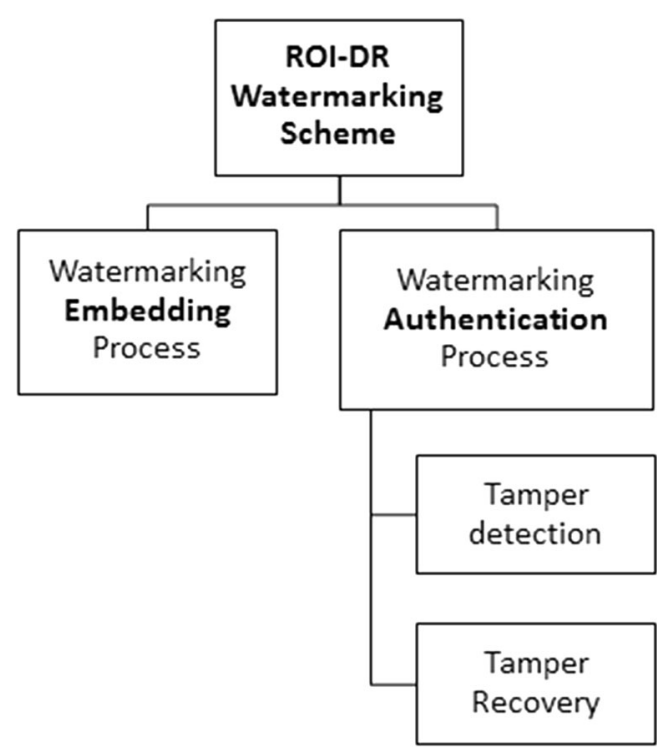

Fig. 1 The basic skeleton of ROI-DR watermarking scheme

medical images excluding the initial configuration and setup time [2]. As the watermarking process is the background work before the diagnosis process starts, the processing time should be shortened in order to minimize waiting time. The time constraint of sequential problem could be resolved in two ways; firstly, optimize or revamp the existing watermarking scheme algorithm to make it lighter and faster on a single frame so it will be faster in multiframes as well. It has been found out that TALLOR watermarking scheme has bundled localization and recovery function into one process, where it localized and recovered the tampered ROI pixel one by one consecutively until the end of ROI pixel [2]; the checking process will be performed until the last ROI pixel even if it is not tampered with, which is time-consuming; therefore, in order to speed up the process, it is proposed to unbundle the tamper localization and recovery function by eliminating the tamper localization features and replace the whole ROI image with the extracted ROI image at the first encounter of the tampered pixel. Secondly, partition the sequential watermarking problem into smaller and manageable parts that can be performed in parallel. This paper will mainly discuss on the first method that is revamping watermarking algorithm on a single frame, whereas the second method is not discussed in this paper. The objective of ROI-based tamper detection and recovery watermarking scheme (ROI-DR) development is actually to solve the time constraint problem of sequential watermarking processing in multiframe medical image environment. A shorter watermarking processing time in a single frame will ultimately speed up the overall sequential watermarking processing time in multiframe environment.

With the technological advancement in communication network, the exchange of medical images between hospitals has become a usual practice nowadays; it has facilitated teleconferences among clinicians and interdisciplinary exchange between clinicians and radiologists for consultative purposes or discussion of diagnostic and therapeutic measures and for distant learning of medical personnel [6]. Thus, medical images are exposed to an open network, where sensitive patient information is vulnerable to hackers' attack. Possible security breaches such as tampering of images to include false data may lead to wrong diagnosis and treatment. Consequently, medical image security has become an important issue that needs to be addressed.

Medical images in different radiological modalities such as X-rays, ultrasounds, and MRI contain vital medical information that can be tampered with easily available image
Fig. 2 ROI and RONI layout for ROI-DR watermarking scheme
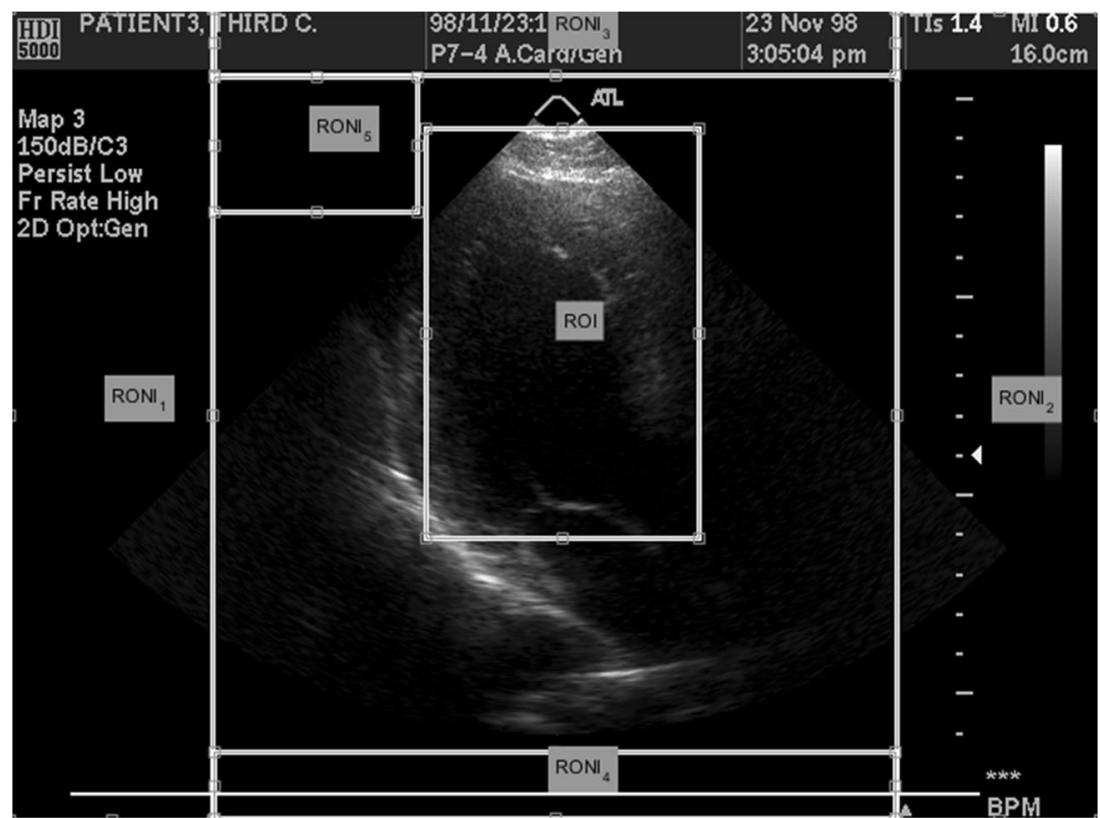
processing tools. Thus, their protection and authentication seem of great importance, and this need will rise along with the future standardization of exchange of data between hospitals or between patients and doctors [7]. The integrity of a medical image can be achieved in three levels [8]:

1. Tamper detection

2. Tamper localization

3. Possible recovery by approximating the tampered region

In order to achieve this along with the requirements of a medical image, a watermark needs to be

1. Fragile and blind

2. Reversible or RONI embedding based

Hence, fragile watermarking helps locate the tampered region with its fundamental property that a watermark becomes invalid for any malicious or unintentional modifications in the watermarked image.

\section{Watermarking Research Based on ROI and RONI}

$\mathrm{ROI}$ is an area of the image where the user is interested and focusing on. Whereas the ROI in medical image is the significant area for clinical diagnosis, thus, any modification on ROI will lead to misdiagnosis, which is not tolerated. In the medical image watermarking, an ROI was defined but untouched and the generated watermark was embedded in the RONI.

Guo and Zhuang suggested three ways to overcome the distortion induced in images by watermark embedding [1]. They are the following:

1. Defining acceptable range of distortion for watermarking.

2. Separating an image into protection zone and insertion zone such as ROI and RONI.

3. Considering watermarking as an invertible manner to recover the original image at the watermark decoder site. Hence, ROI indicates the region significant for diagnosis and other clinical uses, and RONI indicates the complementary region of ROI, which has lesser or almost no significance in diagnosis.

Zain et al. have defined an acceptable range of distortion for watermark embedding through clinical validation, which is expensive [9]. In contrast, separation of ROI and RONI in medical images is not straightforward and may require the interaction/approval of a doctor/radiologist. In addition, making such separation is sometimes very difficult, although it is applied in several watermarking schemes [10, 11].

Coatrieux and Lecornu had identified three kinds of watermarking methods for medical images [12]:

1. Embedding the watermark within the RONI, so it does not affect clinical diagnosis. The ROI is often used for
Table 1 Bit information that embedded into RONI area

\begin{tabular}{|c|c|c|}
\hline $\begin{array}{l}\text { RONI } \\
\text { area }\end{array}$ & $\begin{array}{l}\text { Embedded bit } \\
\text { information }\end{array}$ & Objective \\
\hline 1 and 2 & ROI bits & For ROI recovery process \\
\hline 3 & Hashed ROI value & To verify tampering occurrence \\
\hline 4 & $\begin{array}{l}\text { Hashed compressed } \\
\text { ROI values }\end{array}$ & $\begin{array}{l}\text { To further verify tampering occurrence } \\
\text { at ROI or RONI }\end{array}$ \\
\hline 5 & $\begin{array}{l}\text { Size of compressed } \\
\text { ROI }\end{array}$ & $\begin{array}{l}\text { Used in retrieving compressed ROI bit } \\
\text { process. }\end{array}$ \\
\hline
\end{tabular}

diagnosis rather than the RONI, which is generally in black, or some non-significance areas are gray [13]. Imperceptibility requirement is less strict since the watermark embedding in the RONI causes no interference with the ROI.

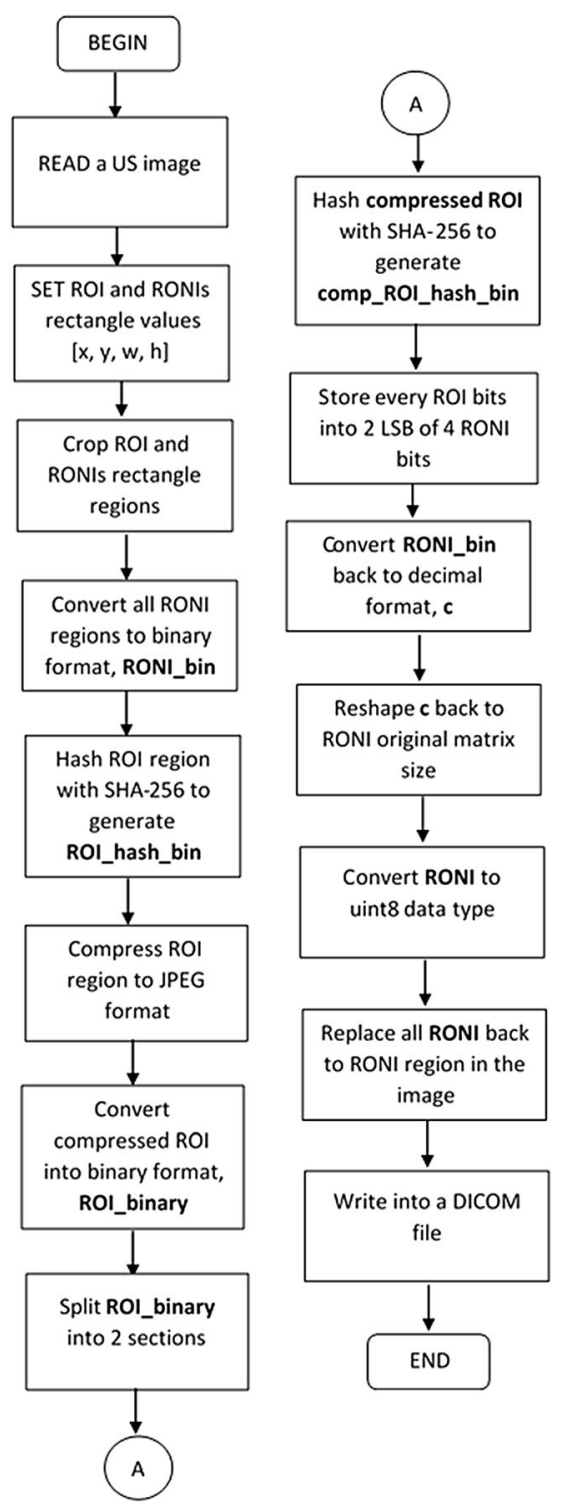

Fig. 3 Program flowchart for ROI-DR watermarking embedding process 
2. Reversible watermarking. The medical image can be restored to its original state upon the removal of the embedded watermark. However, this method often encountered limited storage capacity problem as compared to nonreversible method.

3. Minimizing the distortion caused by watermarking. The watermark replaces some image details such as least significant bits (LSBs) of the image or details lost after lossy image compression.

Sung et al. proposed a dual watermarking scheme by combining robust and fragile watermarking techniques [14]. Robust watermarking is resistant to possible attacks such as image processing. Fragile watermarking can be easily destroyed or undetectable after modification is done on the image. But fragility is an advantage, where it can be used for authentication. The proposed scheme is done by embedding fragile watermark into the result of robust watermarking. In order to prevent interference with the medical image, watermark is embedded by avoiding ROI. The proposed scheme can provide high robustness against median filter attack compared to other attacks.

Research by Fotopoulos et al. [7] had implemented a reversible RONI for brain MRI scans. ROI is defined by using a rectangle that contains the whole head shape and is inserted in the RONI [7]. In addition to other research, the embedding process in the RONI is adaptable. If RONI has enough space, the ROI is compressed in a lossless way, and if not, it is compressed in the best possible way before embedding occurs.

The practicality of watermarked medical images had been tested. Jasni and Abdul performed clinical evaluation of 225 medical images that were embedded with 256bit watermark on RONI and $480 \mathrm{~K}$ bits in both RON and ROI [9]. The results show that watermarking did not alter the clinical diagnosis and is safe in terms of preserving the image quality.

Liew et al. proposed a reversible watermarking scheme (TALLOR) by dividing image into ROI and RONI [2]. ROI is the significant part of the medical images that is used by doctors to diagnose the patients, and RONI is the area outside the ROI. Watermarking for tamper detection and recovery is done in the ROI area based on Jasni's scheme. The original least significant digits (LSBs) that are removed in watermark embedding process are stored in RONI after compression. The stored LSBs later can be used to restore the image to its original bit value, so the watermarking scheme can be reversible. Tamper

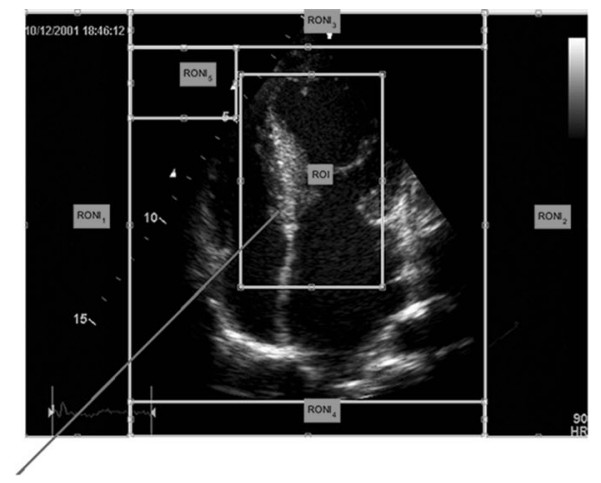

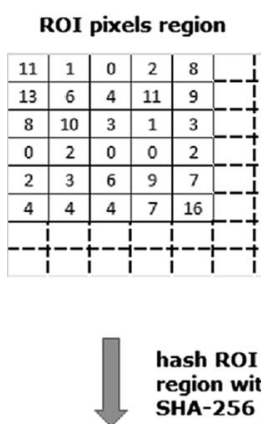

ROI_hash

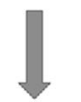

Store in RONI 3

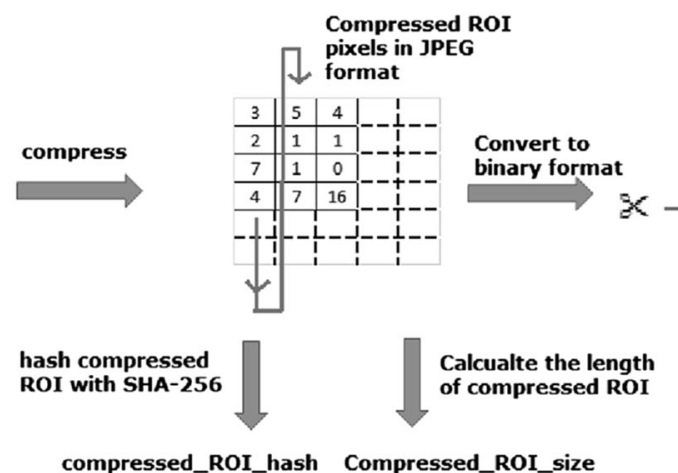

compressed_ROI_hash Compressed_ROI_size

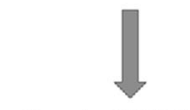

Store in RONI 4

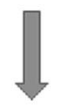

Store in RONI 5

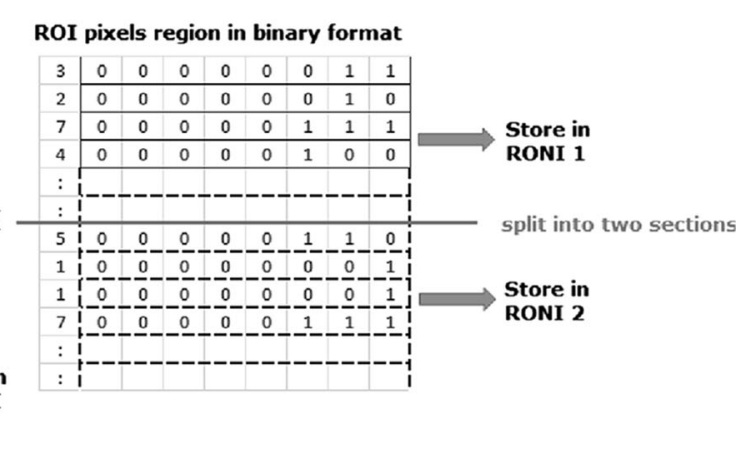

ROI pixels region in binary format

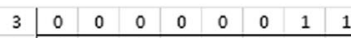

\begin{tabular}{l|l|l|l|l|l|l|l|l|}
2 & 0 & 0 & 0 & 0 & 0 & 0 & 1 & 0 \\
\hline & 0 & 0 & 0 & 0 & 0 & 1 & 1 & 1 \\
\hline
\end{tabular}

7 \begin{tabular}{l|llll|l|l|l|}
\hline & 0 & 0 & 0 & 0 & 1 & 1 & 1 \\
\hline & 0 & 0 & 0 & 0 & 1 & 0 & 0
\end{tabular}$\quad$ Store in

$\begin{array}{lllllllll}0 & 0 & 0 & 0 & 0 & 1 & 1 & 1 \\ 0 & 0 & 0 & 0 & 1 & 0 & 0\end{array}$ RONI

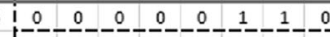

100

$: 1$

Fig. 4 Zoom-in watermarking embedding process 


\section{RONI_1 pixels region in binary format}

RONI_1 pixels region

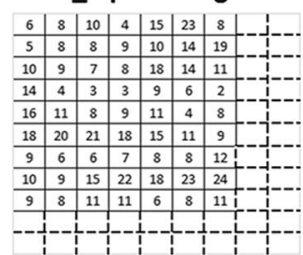

convert

from

decimal to

binary

format

Overwrite

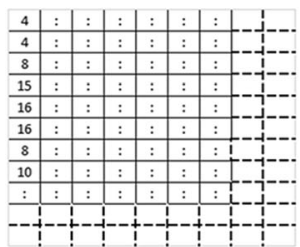

Reshape the RONI 1 decimal matrix image back to its original matrix size in

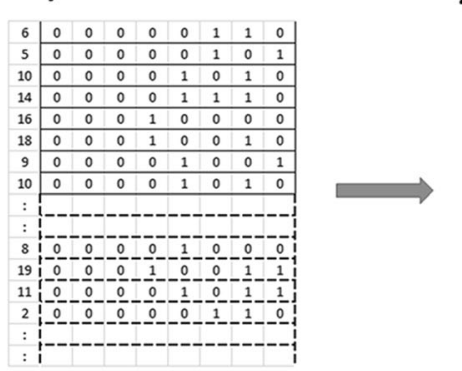

nat

\section{Watermarked RONI_1} pixels region
RONI_1 LSBs are embeded

2 bits from ROI

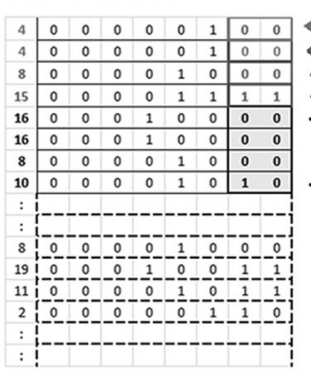

embedding 2 bits

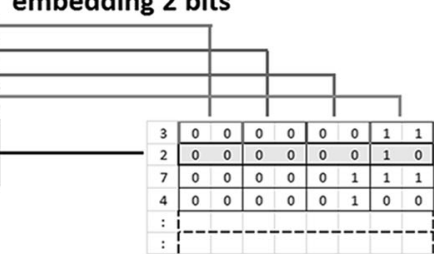

ROI pixels region in binary format

Fig. 5 Watermark embedding into RONI_1 pixels

Localization and Losssless Recovery with ROI Segmentation (TALLOR-RS) is an improved watermarking scheme by further dividing the ROI into segments; each segments needs to be authenticated individually. The authentication can be performed in a multilevel manner where only suspected segments will be examined further for tampering [15].

\section{Background}

ROI-based tamper detection and lossless recovery watermarking scheme (ROI-DR) are basically constituted by two main processes, which are watermarking embedding and authentication process as illustrated in Fig. 1.

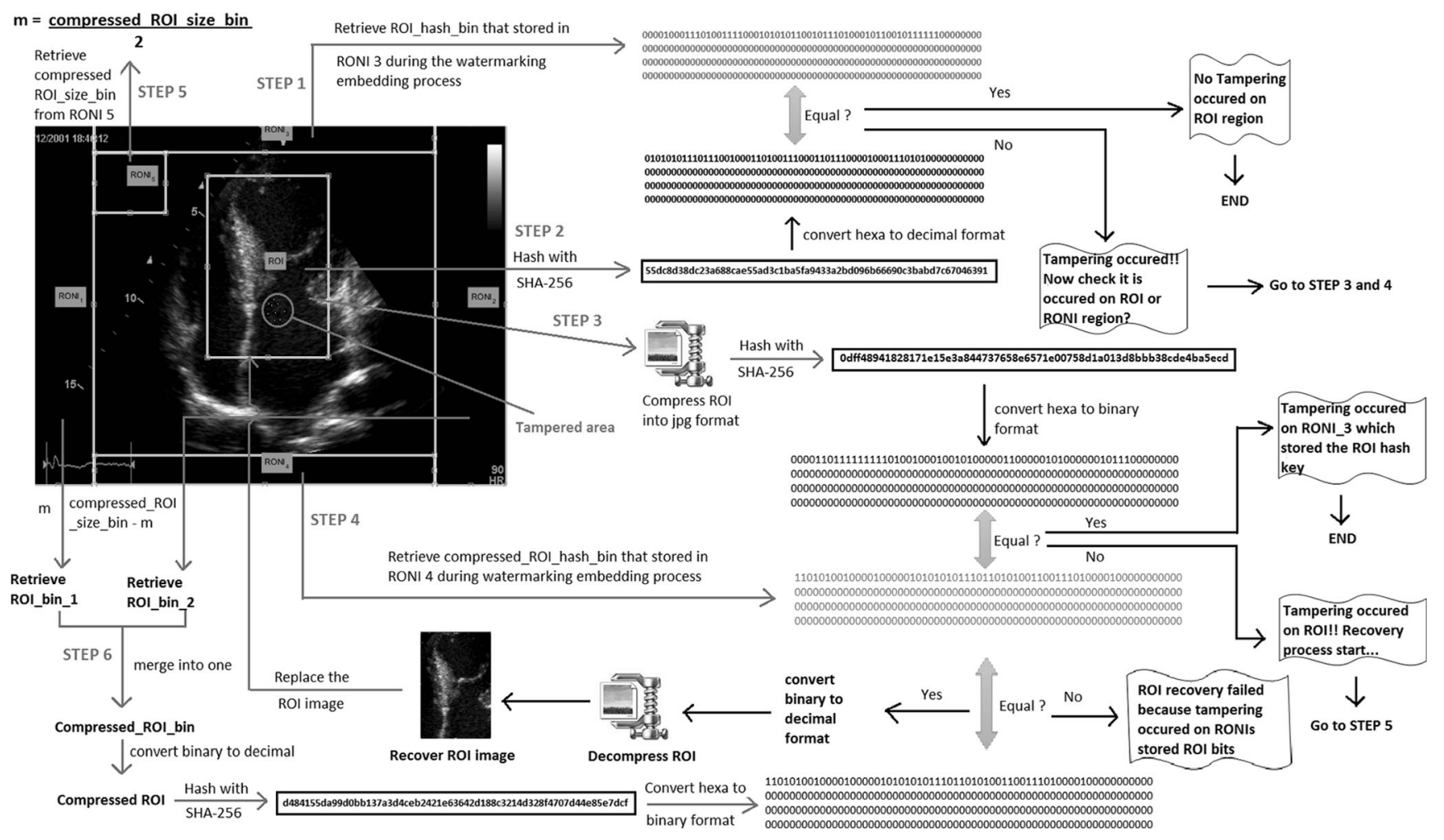

Fig. 6 Detail view on ROI-DR watermarking authentication process 
Fig. 7 Watermarking process flow

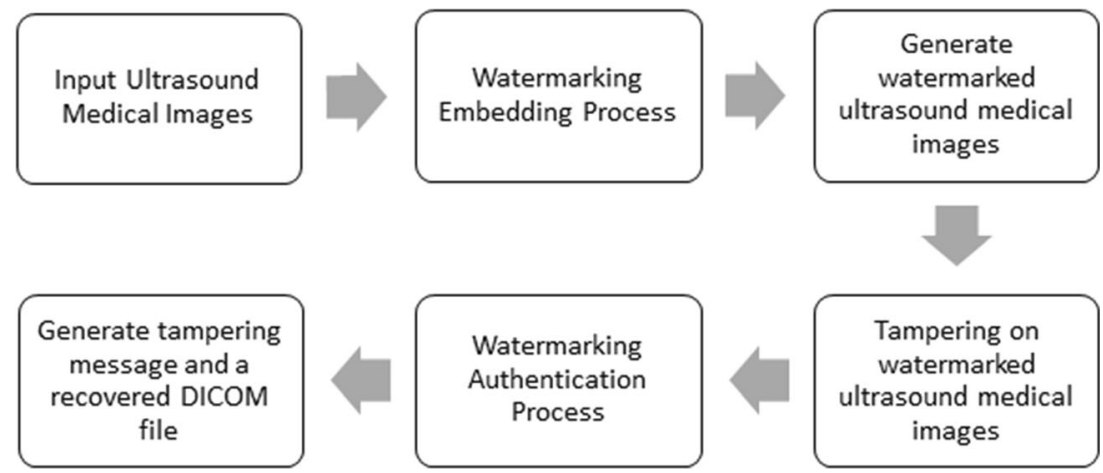

Watermarking embedding process crops a preidentified rectangle image from the center of an ultrasound medical image; this rectangle image is identified as an ROI, a region that has a significant clinical diagnosis value, and any distortion on it should be avoided; in order to leave ROI untouched, the cropped ROI is compressed, converted into bits, and embedded into RONI's LSB and a watermarked medical image is generated. After a period of time, the watermarked medical image will then be used in watermarking authentication process before clinical diagnosis; it is to check whether there is any tampering occurred during that period of time. Watermarking authentication process extracts and decompresses the embedded bits and compares the ROI bits in the medical image; if the result has shown negative, it means that the ROI was tampered, and then, the tamper recovery process is performed to ensure that the medical image is recovered to its original form.

The proposed watermarking scheme, TALLOR and TALLOR-RS [15], have some attributes in common, such as stored ROI bits into RONI's LSB, implemented JPEG compression techniques, and SHA-256 hashing method in the algorithm; therefore, a comparison on these three watermarking schemes will be conducted and speed-up factors of ROI-DR watermarking scheme in relative to TALLOR and TALLOR-RS will be measured. In order to ensure fairness in comparison result, these three watermarking schemes will conduct experiment under the same hardware and software environment and using the same set of ultrasound medical image samples for testing purposes.

\section{Image Preparation}

An ultrasound image is divided into ROI and RONI as shown in Fig. 2. In this scheme, one rectangle is used for the ROI and five rectangles in the RONI. ROI-DR watermarking scheme basically consists of two main process, that is, the watermarking embedding process and watermarking authentication process, which are described in the following.

\section{Watermarking Embedding Process}

Watermarking embedding process is a process of inserting ROI bits into RONI areas of an ultrasound medical image to generate a watermarked ultrasound medical image, which later is used in watermarking authentication process. ROI of ultrasound medical image is identified, cropped, compressed with JPEG, and hashed with SHA-256. The generated compressed ROI and its hashed values are then converted into bits and stored into RONI areas as described in Table 1. The purpose of compression is to reduce the size of ROI embedding bits and thus reduce the watermark payload and the elapsed time for watermarking embedding process. The compressed ROI bits will then split into two parts and embedded into RONI 1 and RONI 2, respectively.

One ROI pixel consists of 8 bits, which is four pairs of bits; each pair of ROI bits is inserted into two LSBs of each RONI, which means that one pixel of ROI will require 4 pixels of RONI for watermarking embedding process. Therefore, it is an essential implement error handling, which is to ensure sufficient RONI storage space for compressed ROI bits and its hashed values before watermarking embedding process. The ROI and RONI layout is illustrated in Fig. 2. A watermarked medical image is produced after the watermarking embedding process, and it is used as an input file of watermarking authentication process before clinical diagnosis.

Table 2 Testing object properties: six sets of ultrasound medical image samples

\begin{tabular}{lll} 
Ultrasound medical images & Image dimension in pixels & Bits per pixel \\
\hline US_1.dcm & $640 \times 480$ & 8 \\
US_2.dcm & $640 \times 480$ & 8 \\
US_3.dcm & $640 \times 476$ & 8 \\
US_4.dcm & $640 \times 480$ & 8 \\
US_5.dcm & $640 \times 476$ & 8 \\
US_6.dcm & $670 \times 480$ & 8 \\
\hline
\end{tabular}


Fig. 8 Ultrasound medical image samples

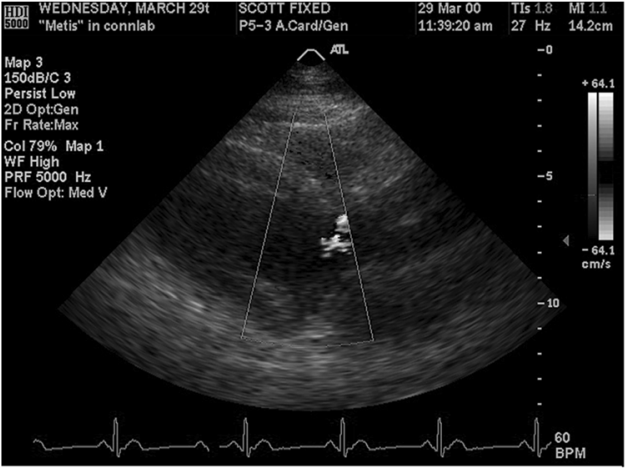

US_1.dcm

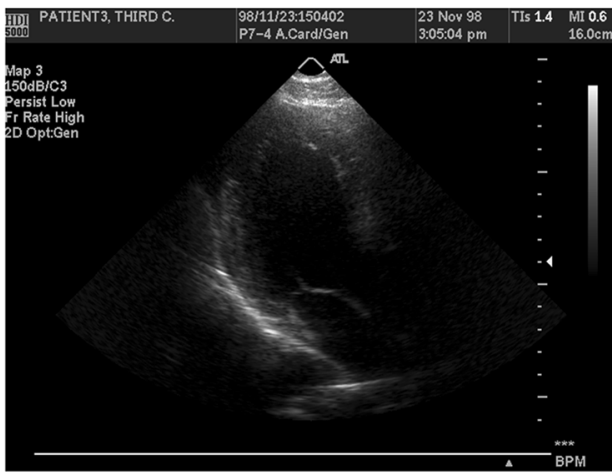

US_3.dcm

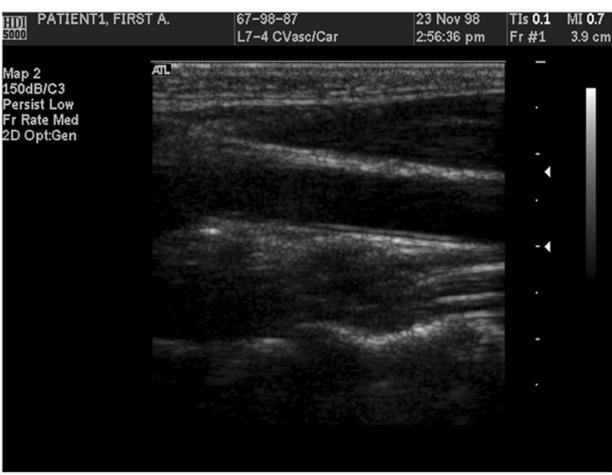

US_5.dcm

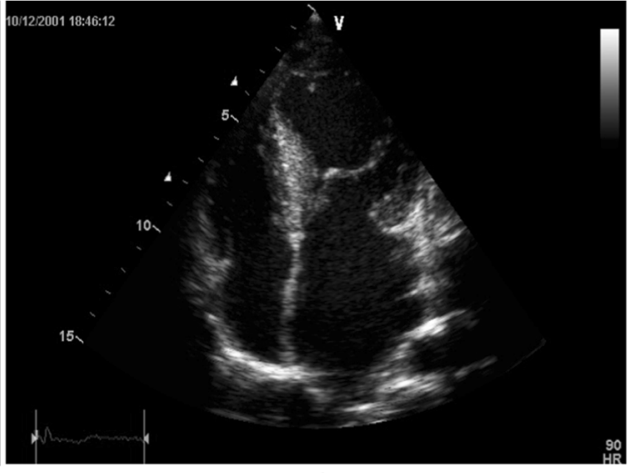

US_2.dcm

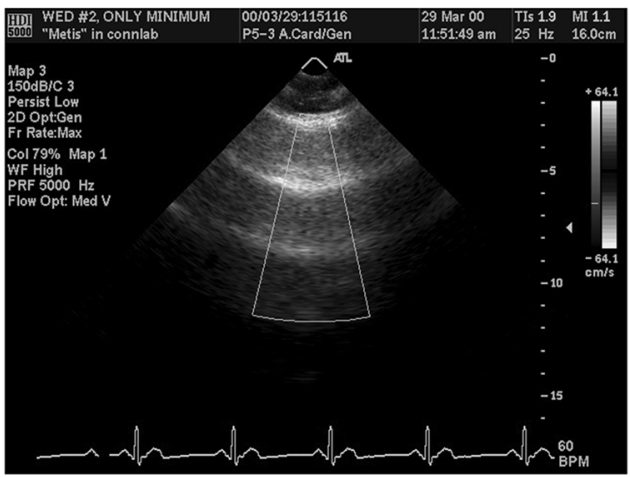

US_4.dem

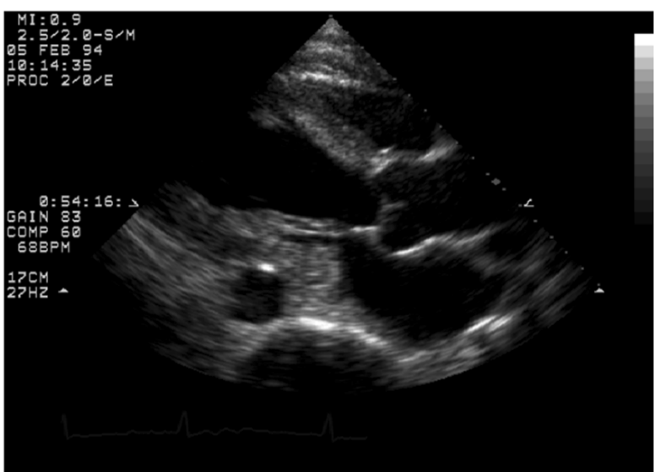

US_6.dcm
Watermarking Authentication Process

Watermarking authentication process is a process to verify whether there is any tampering that occurred at ROI areas and perform recovery process once the tampering is detected. The SHA-256 hashing method has been applied intensively in this process. The hashed values that are retrieved from the RONI are compared with the current hashed values of the examined watermarked medical image; if the hashed ROI bit comparison is positive, then ROI is not tampered, or else, a further verification on tampering occurrence is required, where hashed compressed ROI values are retrieved and compared with the current hashed compressed ROI values of watermarked medical image; if the result is positive, it confirms that the ROI is not tampered but RONI 3 is tampered, if
Table 3 Speed-up factor of TALLOR and TALLOR-RS in relative to ROI-DR watermarking embedding process

\begin{tabular}{lll}
\hline Ultrasound samples & \multicolumn{2}{l}{ Speed-up factor in relative to ROI-DR } \\
\cline { 2 - 3 } & TALLOR & TALLOR-RS \\
\hline US_1 & 25.769 & 28.674 \\
US_2 & 23.257 & 27.512 \\
US_3 & 21.612 & 26.733 \\
US_4 & 24.407 & 25.601 \\
US_5 & 20.387 & 25.989 \\
US_6 & 19.889 & 25.415 \\
Average & 22.554 & 26.654 \\
\hline
\end{tabular}


Table 4 PSNR values of three different watermarking schemes: ROIDR, TALLOR, and TALLOR-RS watermarking embedding process

Watermarking embedding process

\begin{tabular}{|c|c|c|c|c|c|c|}
\hline \multirow[b]{2}{*}{$\begin{array}{l}\text { Ultrasound } \\
\text { samples }\end{array}$} & \multicolumn{2}{|c|}{ ROI-DR } & \multicolumn{2}{|c|}{ TALLOR } & \multicolumn{2}{|c|}{ TALLOR-RS } \\
\hline & $\begin{array}{l}\text { PSNR } \\
(\mathrm{dB})\end{array}$ & $\begin{array}{l}\text { Elapse } \\
\text { time (s) }\end{array}$ & $\begin{array}{l}\text { PSNR } \\
\text { (dB) }\end{array}$ & $\begin{array}{l}\text { Elapse } \\
\text { time (s) }\end{array}$ & $\begin{array}{l}\text { PSNR } \\
\text { (dB) }\end{array}$ & $\begin{array}{l}\text { Elapse } \\
\text { time (s) }\end{array}$ \\
\hline US_1 & 47.953 & 1.024 & 47.944 & 26.375 & 48.179 & 29.348 \\
\hline US_2 & 47.982 & 0.978 & 47.986 & 22.740 & 48.530 & 26.900 \\
\hline US_3 & 48.782 & 0.971 & 48.739 & 21.125 & 48.819 & 25.951 \\
\hline US_4 & 48.107 & 0.978 & 48.265 & 23.871 & 49.547 & 25.038 \\
\hline US_5 & 48.330 & 0.967 & 48.302 & 19.814 & 48.991 & 25.130 \\
\hline US_6 & 49.330 & 0.950 & 49.244 & 18.898 & 49.591 & 24.148 \\
\hline Average & 48.414 & 0.978 & 48.413 & 22.137 & 48.943 & 26.086 \\
\hline
\end{tabular}

the result is negative, it confirms that the ROI is tampered, the recovery process is then performed. Before the recovery process is performed, it is necessary to ensure that RONI 1 or RONI 2 which stored the ROI bits is not tampered; these could be accomplished by comparing the retrieved compressed ROI bits from RONI 1 and RONI 2 with current hashed compressed ROI bits; if both of them are equal, which means RONI 1 and RONI 2 are not tampered, it is safe to perform the ROI recovery process, if both of them are not equal, it confirms that tampering has occurred at RONI 1 or RONI 2, it means that the stored ROI bits have been tampered and could no longer be used to recover ROI as intended.

The following are research methodology for ROI-DR watermarking scheme.

\section{Research Methodology}

The research methodology of ROI-DR watermarking embedding and authentication process will be discussed in sections I and II, respectively.

\section{Watermarking Embedding Process}

Step 1: Set ROI and RONI rectangle values.

Read an ultrasound medical image and set the ROI and region of RONI rectangle values, which are $[x$-coordinate value, $y$-coordinate value, width, height].

Step 2: Crop ROI and RONI rectangle regions.

Crop out the image within the ROI and RONI rectangle regions.

Step 3: Convert all RONI regions to a binary format.

Convert all the cropped RONI regions such as RONI_1 until RONI 5 to a binary format and ready their last two LSBs for ROI bits and hashed value storage.

Step 4: Hash cropped ROI region with SHA-256.

Hash the cropped ROI region with SHA-256 to generate ROI_hash_bin and store into RONI_3 region, in which later will be retrieved and used in watermarking authentication process.

Step 5: Compress ROI region to JPEG format.

Compress the ROI region into JPEG format in order to reduce the size and its payload into RONI storage.

Step 6: Convert the compressed ROI into a binary format.

Compressed ROI will need to be converted into a binary format before embedding bits into the last two LSBs of RONI. The details will be illustrated in Fig. 4.

Step 7: Split ROI_binary into two sections.

Split the ROI_binary (which is generated from step 6) into two sections and store it into RONI_1 and RONI_2, respectively, in step 9.

Step 8: Hash compressed ROI with SHA-256.

Compressed ROIs are hashed with SHA-256 to generate comp_ROI_hash_bin and store it into RONI 4 .

Step 9: Store ROI bits into RONI LSB.

Every ROI pixel consists of 8 bits, in which is
Fig. 9 Ultrasound medical image $\mathbf{a}$ before and $\mathbf{b}$ after watermarking embedding processes

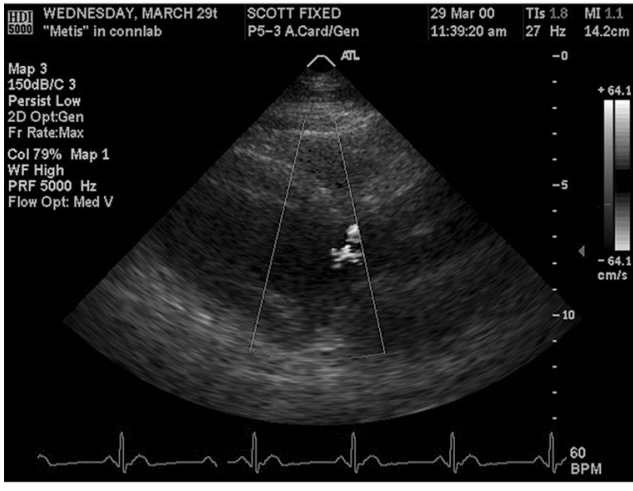

(a) Before watermarking embedding process

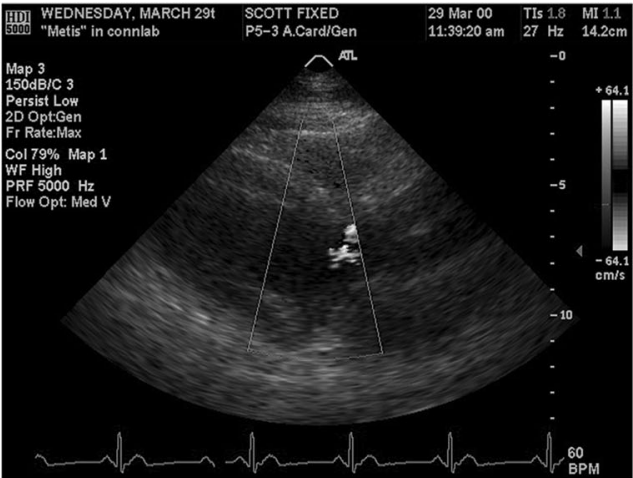

(b) After watermarking embedding process 
Table 5 Test plan for testing the robustness of watermarking towards tampering

\begin{tabular}{ll}
\hline $\begin{array}{l}\text { Test } \\
\text { no. }\end{array}$ & Description \\
\hline 1 & Test on non-tampering watermarked US images \\
2 & $\begin{array}{l}\text { Test on where ROI of US images is tampered in different ways } \\
\text { Comparison elapsed time between TALLOR, TALLOR-RS, and ROI-DR watermarking authentica- } \\
\text { tion processes }\end{array}$ \\
4 & $\begin{array}{l}\text { Test on where RONIs of US image are tampered } \\
\text { Test on where tampering has occurred outside of ROI and RONI rectangle area } \\
6\end{array}$ \\
\hline
\end{tabular}

$4 \times 2$ bits, where each pixel is split into four pairs of bits, and store each pair of bits into the two LSBs of each RONI pixel. In summary, one ROI pixel will require four RONI pixels for storage. The details are illustrated in Fig. 5.

Step 10: Convert RONI_bin back to decimal format, $c$. RONI_bin is converted back to a decimal format as variable $c$ after all ROI bits and hash values have been stored into RONI_bin.

Step 11: Reshape $c$ back to RONI original matrix size.

Reshape variable $c$ from a single column matrix into RONI original matrix size.

Step 12: Convert RONI to uint8 data type.

Convert RONI from double to uint8 data type.

Step 13: Replace all RONI back to the RONI region in the image.

Replace all bits into embedded RONIs (watermark) back to the RONI region in the image, in which a watermarked ultrasound medical image is formed.

Step 14: Write into a DICOM file.

Lastly, write the watermarked ultrasound image into a DICOM file. The process described previously is shown in Fig. 3, and the details are illustrated in Figs. 4 and 5.

\section{Watermarking Authentication Process}

The following are algorithm flows of watermarking authentication process.

\section{Configuration Setting}

Configuration setting is similar to step 1 to step 4 in ROI-DR watermarking embedding process.

\section{Authentication Process}

Step 1: Retrieve ROI_hash_bin from RONI 3.

Retrieve ROI_hash_bin that stored RONI 3 during the watermarking embedding process.

Step 2: Hash ROI with SHA-256 to generate ROI_hash_bin and compare it with step 1.

If ROI_hash_bin retrieved is equal to the ROI hash_bin generated, then it means that no tampering has occurred on ROI region and terminates the authentication process, or else, it means tampering has occurred and a further verification is required to check whether it has occurred on ROI or RONI; hence, proceed to the next step.

Table 6 Test cases for test no. 1: test on non-tampering watermarked US images

\begin{tabular}{llll}
\hline Test data & Elapsed time (s) & Expected result & Actual result \\
\hline Watermarked US_1 & 0.0490 & Display “ROI hash is equal; therefore, no tampering occurred!!” message & Same as expected result \\
Watermarked US_2 & 0.1085 & Display “ROI hash is equal; therefore, no tampering occurred!!” message & Same as expected result \\
Watermarked US_3 & 0.1154 & Display “ROI hash is equal; therefore, no tampering occurred!!” message & Same as expected result \\
Watermarked US_4 & 0.0585 & Display “ROI hash is equal; therefore, no tampering occurred!!” message & Same as expected result \\
Watermarked US_5 & 0.1062 & Display "ROI hash is equal; therefore, no tampering occurred!!” message & Same as expected result \\
Watermarked US_6 & 0.0421 & Display "ROI hash is equal; therefore, no tampering occurred!!” message & Same as expected result \\
\hline
\end{tabular}

Conclusion: tested successfully 
Table 7 Test cases for test no. 2: test on where ROIs of US images are tampered in different ways

\begin{tabular}{|c|c|c|c|c|}
\hline $\begin{array}{l}\text { ROIs of US images are tampered in } \\
\text { different ways as indicated below }\end{array}$ & Elapsed time (s) & Expected result & Actual Result & $\begin{array}{l}\text { ROI recovery } \\
\text { measured in PSNR }\end{array}$ \\
\hline $\begin{array}{l}\text { Cloning a portion of image into ROI } \\
\text { (as shown in Fig. 10) }\end{array}$ & 0.4623 & $\begin{array}{l}\text { Display ROI tampered message and } \\
\text { produced a recovered DICOM file }\end{array}$ & Same as expected result & Infinity \\
\hline $\begin{array}{l}\text { Adding salt-and-pepper noise into ROI } \\
\text { (as shown in Fig. 11) }\end{array}$ & 0.4713 & $\begin{array}{l}\text { Display ROI tampered message and } \\
\text { produced a recovered DICOM file }\end{array}$ & Same as expected result & Infinity \\
\hline $\begin{array}{l}\text { Flipping a portion of image in ROI } \\
\text { vertically (as shown in Fig. 12) }\end{array}$ & 0.3915 & $\begin{array}{l}\text { Display ROI tampered message and } \\
\text { produced a recovered DICOM file }\end{array}$ & Same as expected result & Infinity \\
\hline $\begin{array}{l}\text { Smoothening some areas in ROI } \\
\text { (as shown in Fig. 13) }\end{array}$ & 0.3343 & $\begin{array}{l}\text { Display ROI tampered message and } \\
\text { produced a recovered DICOM file }\end{array}$ & Same as expected result & Infinity \\
\hline
\end{tabular}

Conclusion: tested successfully. Exact ROI recovery produced an infinity result in PSNR

\section{Verification Process on Checking Whether Tampering Has Occurred on ROI or RONI}

Step 3: Generate hash_compressed_roi.

ROI is compressed into JPEG and converted into a binary format and then hashed with SHA-256 to generate hash_compressed_roi.

Step 4: Retrieve compressed_ROI_hash_bin from RONI 4 and compare it with step 3 .
Retrieve compressed_ROI_hash_bin that is stored in RONI 4 during the watermarking embedding process. If compressed_ROI_hash_bin retrieved is equal to hash_compressed_roi generated in step 3, then it means that no tampering has occurred on ROI, but it occurred on RONI 3 region instead, if both values are not equal, it is confirms that tampering has occurred on the ROI region, a further investigation is required to ensure that

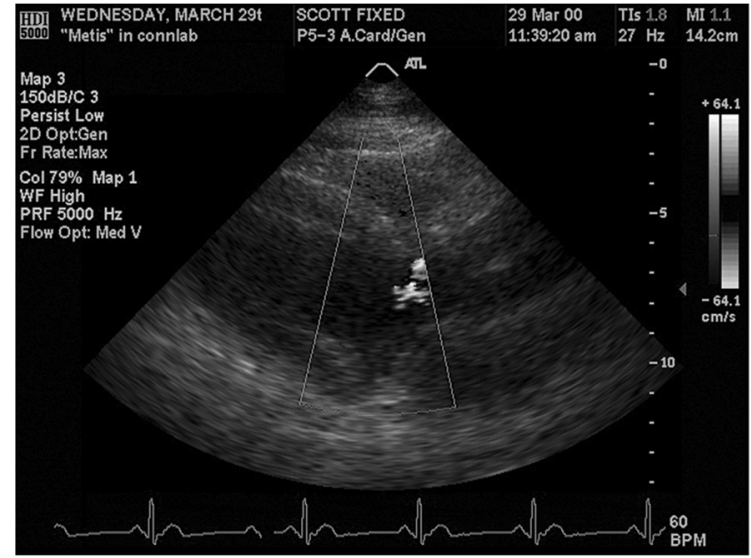

Watermarked_US_1 before tampering

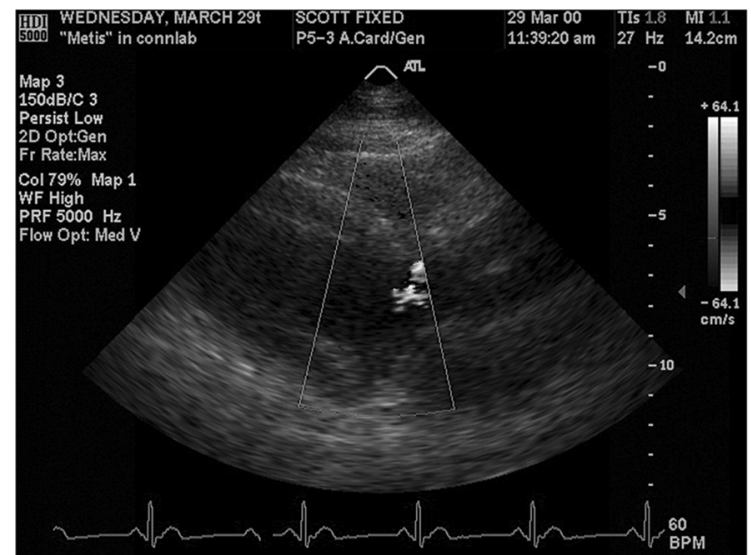

Recovered_US_1.dcm

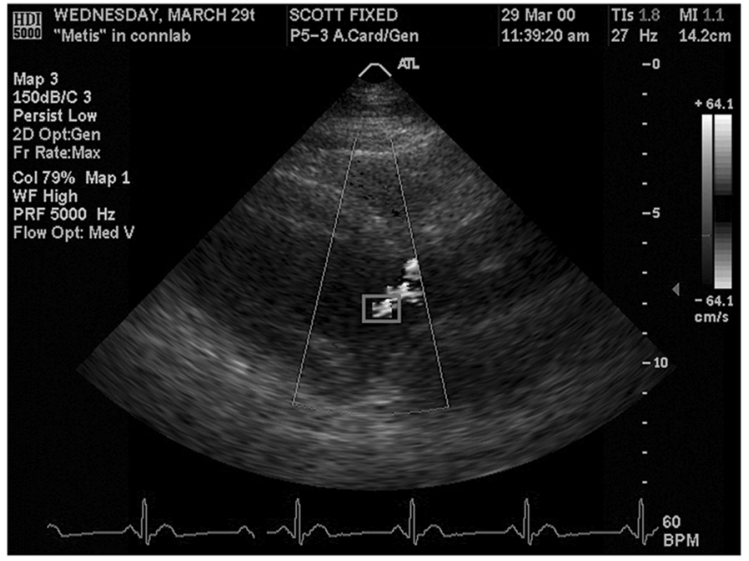

Watermaked_US_1 after tampered by cloning a portion of image into ROI

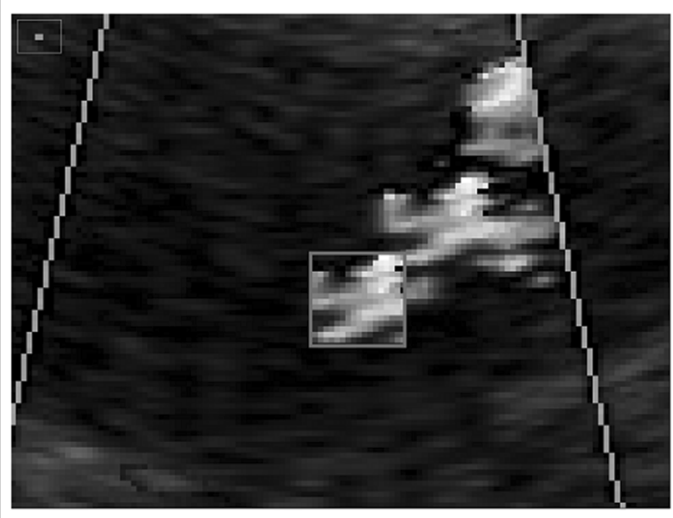

Zoom with $800 \%$

Fig. 10 Ultrasound medical images before and after being tampered by cloning a portion of image into ROI and the recovered ultrasound medical image 


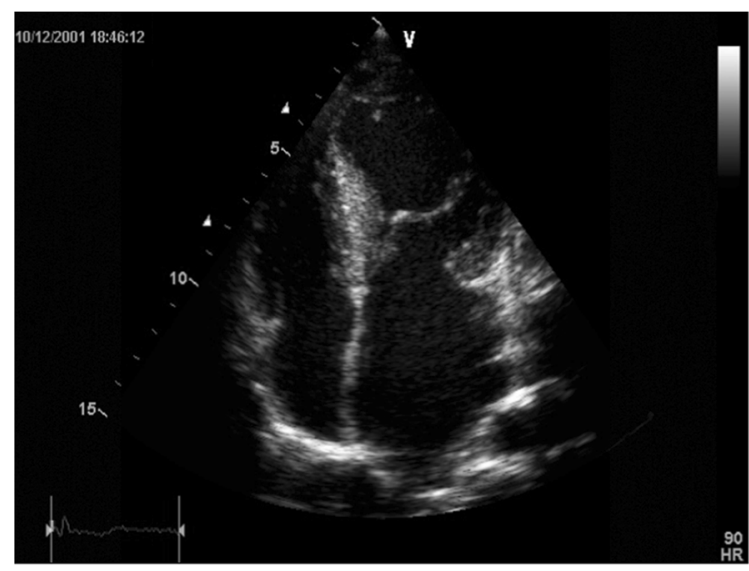

Watermarked_US_2 before tampering

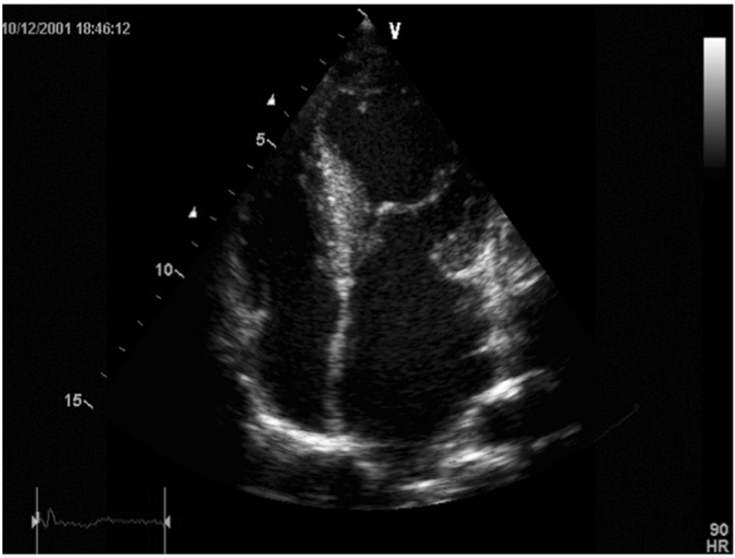

Recovered_US_2.dcm

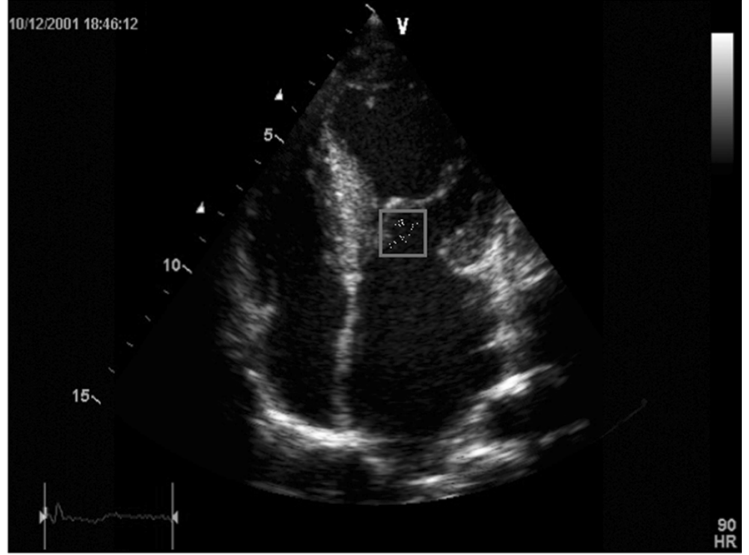

Watermarked_US_2 after tampered by adding "salt and pepper" noise into ROI

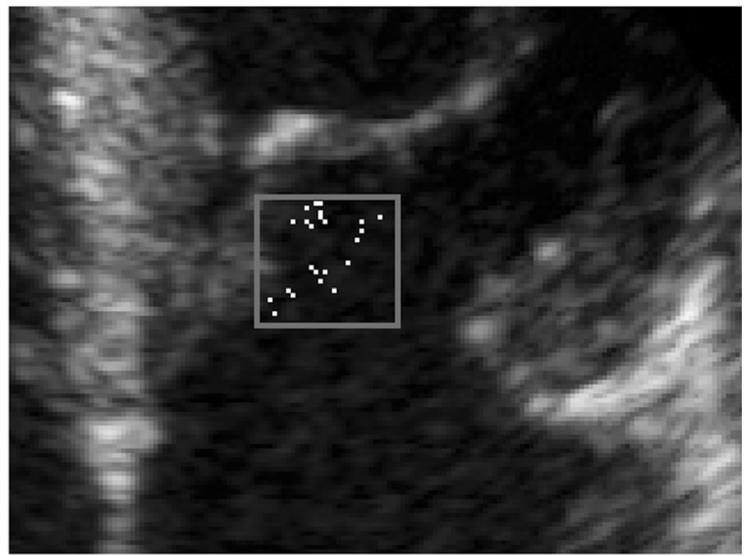

Zoom in the tampered area

Fig. 11 Ultrasound medical images before and after being tampered by adding salt-and-pepper noise into ROI and the recovered ultrasound medical image

tampering has not occurred on RONIs that stored ROI bits before proceeding to ROI recovery, which leads to step 5 .

\section{A Process That Ensures That Tampering Has Not Occurred on RONIs That Stored ROI Bits Before Proceeding to ROI Recovery}

Step 5: Get the halve values of compressed roi_size, $m$. Retrieve compressed ROI_size bin from RONI 5 and divide it to half to generate $m$.

Step 6: Compare compressed_ROI_hash_bin_2 with the values retrieved from step 4 .

Retrieve ROI bin 1 and ROI bin 2 from RONI 1 and RONI 2, respectively, based on $m$ values, and then, merge them into one binary form, compressed ROI 2, and hash it with SHA-256 to generate compressed ROI hash bin 2 , in which it is used to compare with values retrieved from RONI 4 ; if the result is equal, then proceed to the ROI recovery process, or else, it means that tampering has occurred on RONIs which stored ROI bits; thus, no recovery could be done.

\section{A Process That Recovers the ROI Region}

Decompress the compressed_ROI_2 and reshape it according to ROI region size, then replace it into ROI region, and lastly write the whole image into a DICOM file. The process described previously is illustrated in Fig. 6.

\section{Experimental Design}

A general watermarking embedding and authentication process flow (as illustrated in Fig. 7) has provided a perspective on digital watermarking experimental design. The experiment will focus on watermarking embedding and authentication process. Six samples of ultrasound medical images in DICOM format will be prepared as input files, and peak signal-to-noise ratio (PSNR) 



Watermarked_US_3 before tampering

Watermarked_US_3 after tampered by flipping a portion of image in ROI vertically

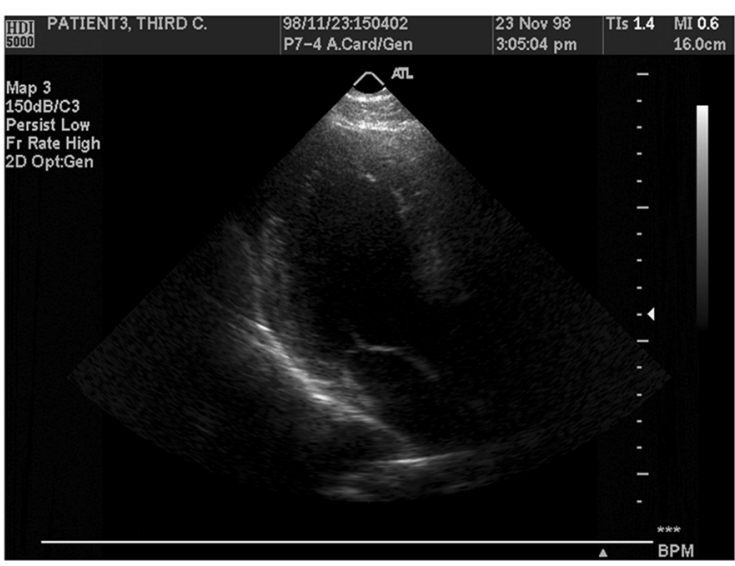

Recovered_US_3.dcm

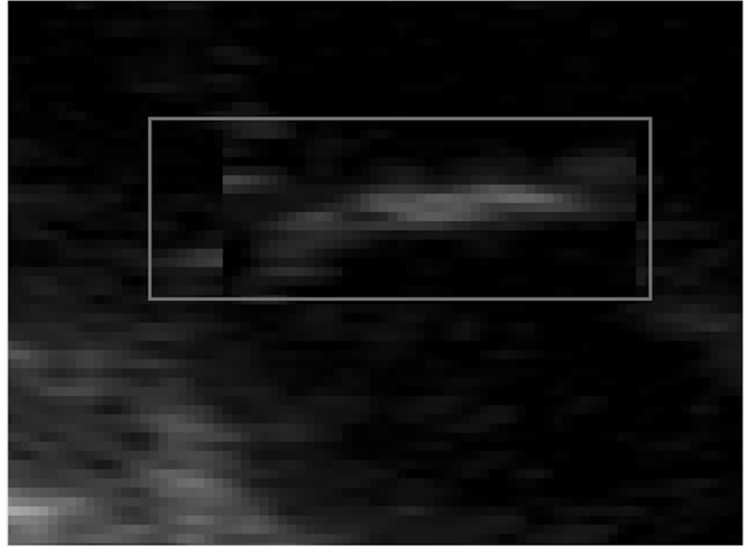

Zoom in the tampered vertical flip area

Fig. 12 Ultrasound medical images before and after being tampered by flipping a portion of image in ROI vertically and the recovered ultrasound medical image

is used to measure the integrity of watermarked ultrasound medical images generated; the higher PSNR values reflected the better image integrity, which means less distortion in image. Several tampering methods were applied on the watermarked ultrasound medical images generated, such as cloning a portion of image into the ROI area, adding salt-and-pepper noise into the ROI area, smoothening certain areas of ROI, flipping a portion of image vertically, and filling a portion of images with a black color. Tampering will be applied at (1) ROI areas, (2) RONIs areas, (3) both ROI and RONI areas, and (4) outside ROI and RONI areas, for testing purposes. All of this tampering will change image pixel values and is detected by the watermarking authentication process, which will then display a message if there is any tampering that occurred. As a result, ROI will be restored and will generate a recovered DICOM file, in which ROI recovery will be tested by PSNR values on ROI areas. Elapsed time will be measured and used to make a comparison with previous research, TALLOR and TALLOR-RS [15; it is to prove that the current research, ROI-DR watermarking scheme, has a better performance than TALLOR and TALLOR-RS.
Three important performance metrics were studied. These are the following:

1. Imperceptibility - this is to test the quality of medical images in terms of invisibility of watermarking.

2. Elapsed time - the time taken to perform watermarking embedding and authentication process on medical images.

3. Robustness to tampering - this is to test the effectiveness and efficiency of the tamper detection and recovery function.

A comparison on elapsed time of the current research (ROIDR watermarking scheme) with previous research, TALLOR and TALLOR-RS, has been done under the same environment and conditions, such as same set of ultrasound medical image sources, same ROI size $(160 \times 240$ pixels $)$, same tampered areas in ROI, and same testing environment. It is to ensure fairness in comparison. The main differences between ROI-DR, TALLOR, and TALLOR-RS are RONI layout and organization, the way of storing ROI bits into RONI LSB, and its recovery method. 


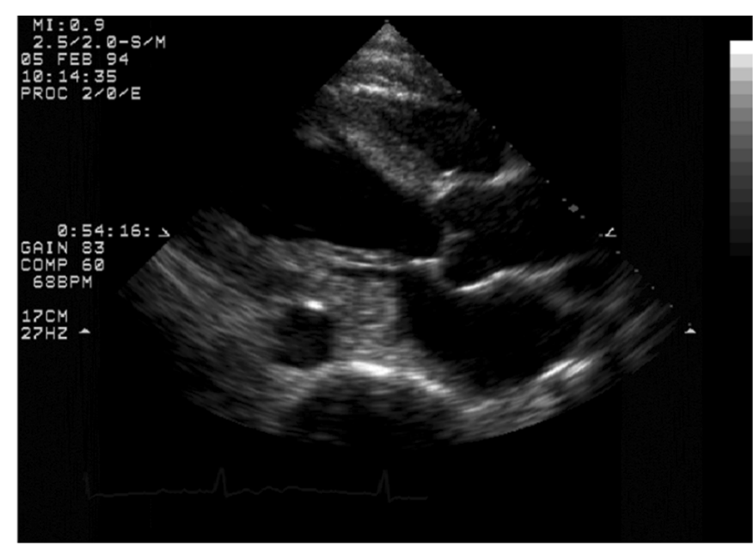

Watermarked_US_6 before tampering

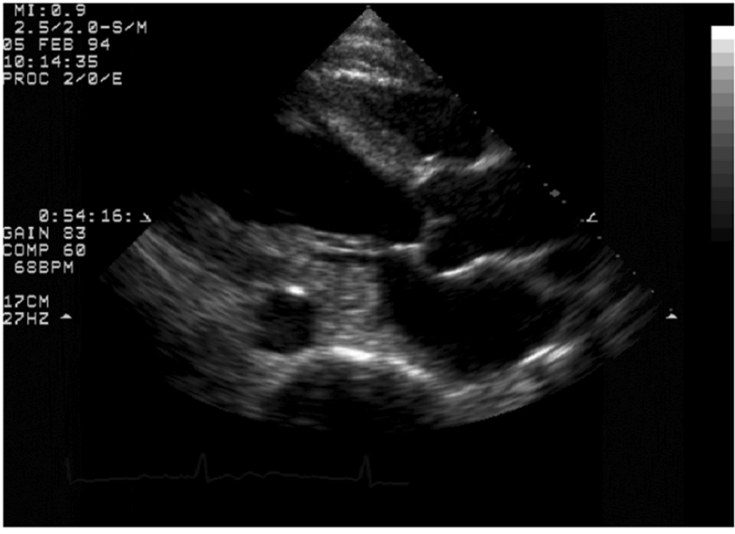

Recovered_US_6.dcm

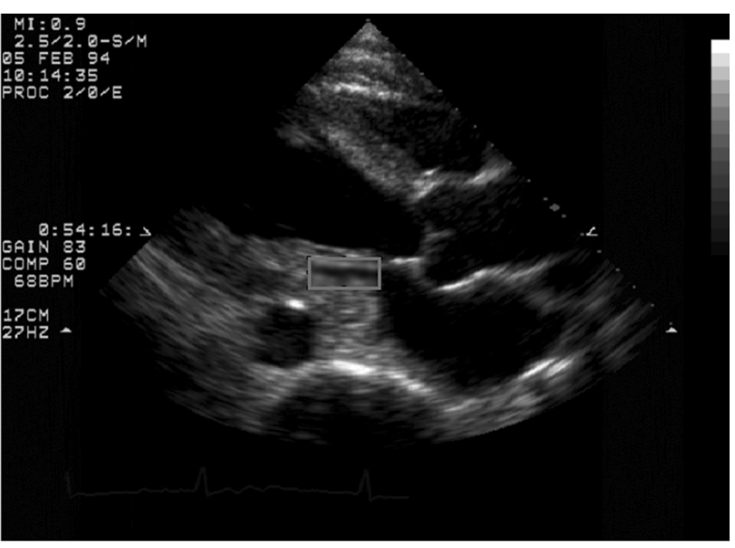

Watermarked_US_6 after tampered by smoothening some areas in the ROI

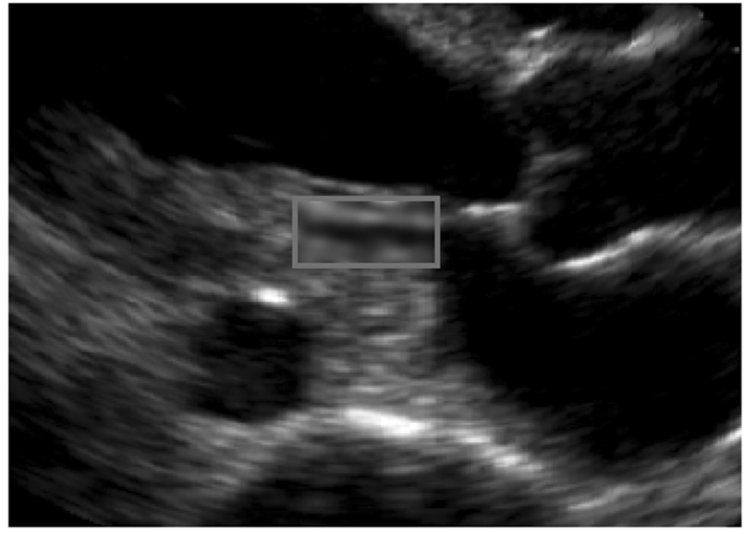

Zoom in the tampered area

Fig. 13 Ultrasound medical images before and after being tampered by smoothening some areas in ROI and the recovered ultrasound medical image

\section{Imperceptibility}

The perceptibility of a watermarked image can be judged according to its fidelity and quality. Fidelity measures the similarity between images before and after watermarking [16]. High fidelity means that watermarked image is very similar to the original image. The mean square error (MSE) and PSNR were calculated by comparing the watermarked image and original image. Watermarked images may bear visible or invisible distortion due to the embedding process. One way to quantify distortion is through the MSE. This is defined as follows:

$$
\mathrm{MSE}=\frac{1}{n} \sum_{i}^{n}\left(I_{i}^{\prime}-I_{i}\right)^{2}
$$

Table 8 Test cases for test no. 3: comparison of elapsed time between TALLOR, TALLOR$\mathrm{RS}$, and ROI-DR watermarking authentication processes

\begin{tabular}{llcc}
\hline Test data & \multicolumn{2}{l}{ Elapsed time (s) } & \\
\cline { 2 - 4 } & ROI-DR & TALLOR & TALLOR-RS \\
\hline Non-tampering ROI & 0.049 & 1.342 & 7.275 \\
Cloning a portion of image into ROI & 0.462 & 10.421 & 7.232 \\
Adding salt-and-pepper noise into ROI & 0.471 & 9.033 & 6.754 \\
Flipping a portion of image in ROI vertically & 0.392 & 8.268 & 6.597 \\
Smoothening some areas in ROI & 0.334 & 6.427 & 6.222 \\
Average & 0.342 & 7.098 & 6.816 \\
\hline
\end{tabular}


Table 9 Speed-up factor of TALLOR and TALLOR-RS in relative to ROI-DR

\begin{tabular}{llc}
\hline Test data & \multicolumn{2}{l}{ Speed-up factor in relative to ROI-DR } \\
\cline { 2 - 3 } & TALLOR & TALLOR-RS \\
\hline Non-tampering ROI & 27.390 & 148.527 \\
Cloning a portion of image into ROI & 22.543 & 15.645 \\
Adding salt-and-pepper noise into ROI & 19.166 & 14.331 \\
Flipping a portion of image in ROI vertically & 21.118 & 16.849 \\
Smoothening some areas in ROI & 19.227 & 18.614 \\
Average & 21.889 & 42.793 \\
\hline
\end{tabular}

which is the average term by term difference between the original image, $I$, and the watermarked image, $I^{\prime}$. If $I$ and $I^{\prime}$ are identical, then $\operatorname{MSE}\left(I^{\prime}, I\right)=0$. A related distortion measure is the PSNR, measured in decibels $(\mathrm{dB})$. The problem with MSE is that it depends strongly on the image intensity scaling, while PSNR rectifies this problem by scaling the MSE according to the image range [17]. PSNR is defined as follows:

$\operatorname{PSNR}(\mathrm{dB})=10 \log _{10} \frac{\max I^{2}}{\mathrm{MSE}^{\prime}}$

where max $I$ is the peak value of the original image. If the signals are identical, then PSNR is equal to infinity. A high PSNR represents a high fidelity of a watermarked image. In this study, PSNR is used as a measurement for image fidelity. A highquality watermarked image does not have any obvious noticeable distortion caused by the watermark embedding process. The assessment of quality is usually evaluated by human observers and is influenced by personal preferences, which are subjective in nature.

\section{Elapsed Time}

Elapsed time is the main focus of this experiment, and ROI-DR watermarking scheme has been compared with TALLOR and TALLOR-RS in terms of its processing time. These three watermarking schemes have been conducted at the same experimental environment, such as same ultrasound samples and hardware and software testing environment. The result of the total elapsed time used in these three schemes will be collected, and a speed-up factor of TALLOR and TALLOR-RS in relative to ROI-DR scheme will be calculated. A speed-up factor is defined as follows:

Speed up $=\frac{\text { Elapsed time gain in TALLOR or TALLOR-RS watermarking process }}{\text { Elapsed time gain in ROI-DR watermarking process }}$

Table 10 Test cases for test no. 4: test on where RONIs of US image are tampered

\begin{tabular}{|c|c|c|c|c|}
\hline \multirow[t]{2}{*}{ Test data } & \multirow[t]{2}{*}{ Elapsed time (s) } & \multicolumn{2}{|l|}{ Expected result } & \multirow[t]{2}{*}{ Actual result } \\
\hline & & Message display & $\begin{array}{l}\text { Produced } \\
\text { recovered file? }\end{array}$ & \\
\hline $\begin{array}{l}\text { Tampered on RONI } 1 \\
\text { (as shown in Fig. 14) }\end{array}$ & 0.054 & ROI hash is equal; therefore, no tampering occurred!! & No & Same as expected result \\
\hline $\begin{array}{l}\text { Tampered on RONI } 2 \\
\text { (as shown in Fig. 14) }\end{array}$ & 0.118 & ROI hash is equal; therefore, no tampering occurred!! & No & Same as expected result \\
\hline $\begin{array}{l}\text { Tampered on right side of } \\
\text { RONI 3(as shown in Fig. 15) }\end{array}$ & 0.142 & ROI hash is equal; therefore, no tampering occurred!! & No & Same as expected result \\
\hline $\begin{array}{l}\text { Tampered on left side of } \\
\text { RONI } 3 \text { (as shown in Fig. 15) }\end{array}$ & 0.130 & $\begin{array}{l}\text { No tampering has occurred on ROI but it occurred on } \\
\text { RONI_3 instead, in which stored the ROI hash } \\
\text { key!!! }\end{array}$ & No & Same as expected result \\
\hline $\begin{array}{l}\text { Tampered on RONI } 4 \\
\text { (as shown in Fig. 16) }\end{array}$ & 0.060 & ROI hash is equal; therefore, no tampering occurred!! & No & Same as expected result \\
\hline $\begin{array}{l}\text { Tampered on RONI } 5 \\
\text { (as shown in Fig. 16) }\end{array}$ & 0.050 & ROI hash is equal; therefore, no Tampering occurred!! & No & Same as expected result \\
\hline
\end{tabular}


Table 11 Test cases for test no. 5: test on where tampering has occurred outside of ROI and RONI rectangle area

\begin{tabular}{lllll}
\hline Test data & $\begin{array}{c}\text { Elapsed } \\
\text { time }(\mathrm{s})\end{array}$ & Expected result & \multicolumn{1}{c}{$\begin{array}{c}\text { Actual } \\
\text { result }\end{array}$} \\
\cline { 3 - 4 } & & Message display & $\begin{array}{l}\text { Produced } \\
\text { recovered } \\
\text { file? }\end{array}$ & \\
\hline $\begin{array}{c}\text { Tampered at } \\
\text { outside the } \\
\text { area of ROI } \\
\text { and RONI }\end{array}$ & 0.429 & $\begin{array}{c}\text { ROI hash is equal; } \\
\text { therefore, no } \\
\text { tampering } \\
\text { occurred!! }\end{array}$ & $\begin{array}{c}\text { Same as } \\
\text { ex- } \\
\text { pected } \\
\text { result }\end{array}$ \\
\hline
\end{tabular}

\section{Robustness to Tampering}

In order to demonstrate the tamper localization function in detecting forgery, counterfeited images were created by manually modifying the pixel values in the watermarked images using image processing software-ImageJ 1.46r. Six sets of watermarked ultrasound medical images were tampered in different manners such as cloning a portion of image into ROI, adding "salt-and-pepper" noise into ROI and RONI, flipping a portion of image in ROI vertically, and smoothening some areas in ROI.

Different sets of tampered watermarked ultrasound medical images have been used to test the effectiveness and efficiency of the tamper detection and recovery function in multiframe environment. The function's effectiveness was measured by checking whether it could detect and determine the tampering area and able to recover ROI to its original form. The function's efficiency was measured by calculating the speed-up factor, which should be above the threshold value, which means more than 1 , where the elapsed time gain in ROI-DR watermarking scheme must lesser than TALLOR and TALLOR-RS in order to achieve the efficiency testing requirement. Both effectiveness and efficiency testing was performed while testing the function's robustness to tampering. A test plan and test cases have been derived for more systematic robustness testing towards tampering.

\section{Experimental Setup}

The evaluation was performed by running the MATLAB program on a standalone computer with quad-core CPU of Intel i7-4790 CPU @ $3.60 \mathrm{GHz}$ and $3601 \mathrm{MHz}$, with RAM of 4 GB. Six sets of ultrasound medical image samples in DICOM format have been used in this experiment, and their properties are shown in Table 2 and Fig. 8.

\section{Experimental Results and Analysis}

\section{Imperceptibility and Elapsed Time in Watermarking Embedding Process}

This experiment has shown that the ROI-DR has a better performance in elapsed time, where the average speed-up factors are 22.554 and 26.654 for TALLOR and TALLOR-RS, respectively, in relative to ROI-DR watermarking embedding process (as shown in Table 3). The PSNR values are varied based on the RONI LSB which stored the ROI bits; the more coincidence of same value in ROI bits and RONI LSB values, the higher are the PSNR values. Table 4 shows that the average PSNR values of all three watermarking schemes are similar (48.41 48.94 dB) and have fulfilled the imperceptibility requirement, where ultrasound medical images before and after the watermarking process are visually undisguisable (as shown in Fig. 9)

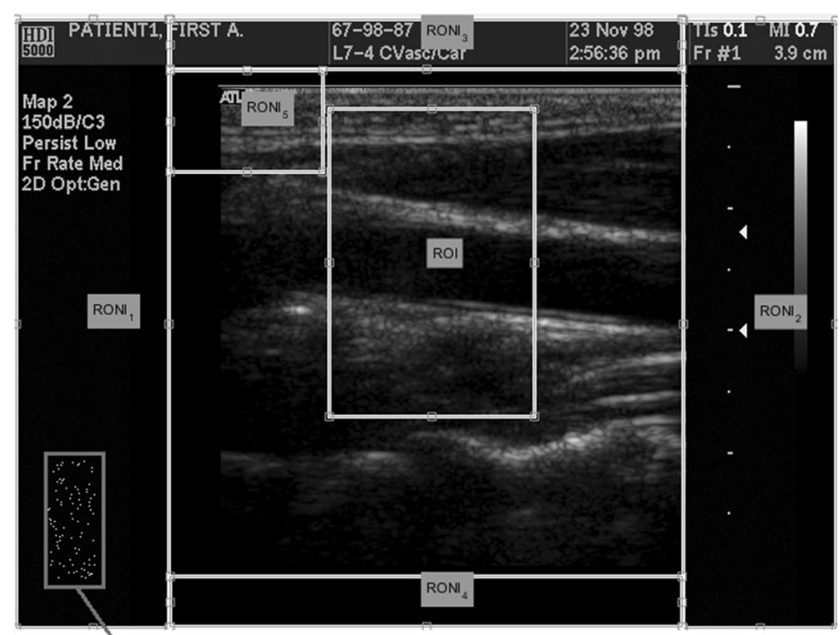

Tampered with salt and pepper in RONI 1

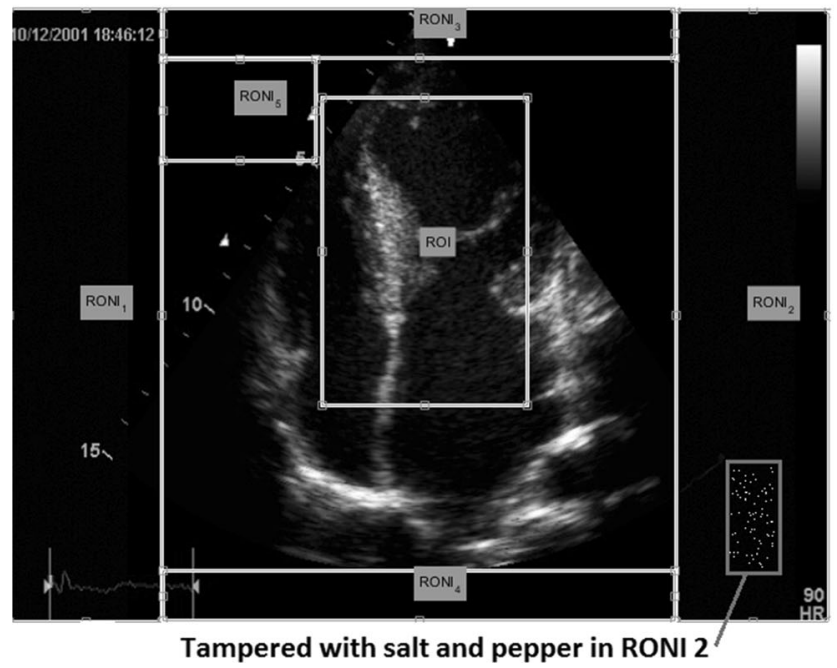

Fig. 14 Ultrasound medical images tampered on RONI 1 and RONI 2 
Fig. 15 Ultrasound medical images tampered on RONI 3
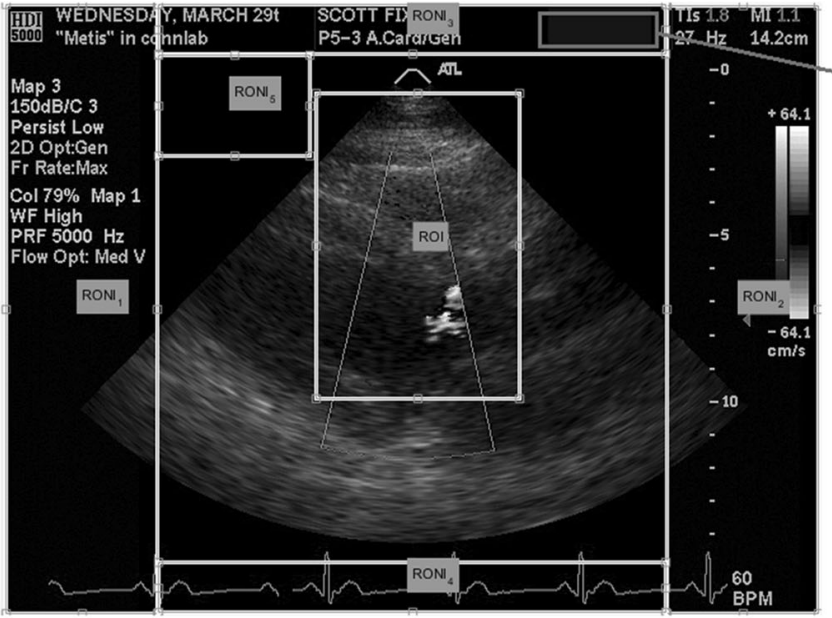

Tampered at the right side of RONI 3 rectangle
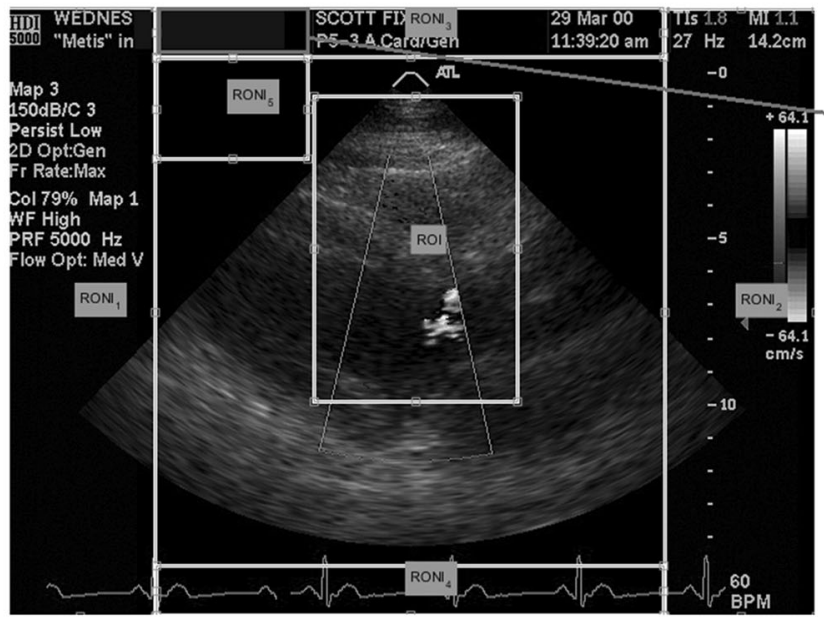

Tampered at the left side of RONI 3 rectangle

\section{Robustness to Tampering and Elapsed Time in ROI-DR Watermarking Authentication Process}

A test plan for testing the robustness of watermarking towards tampering is shown in Table 5. Test cases for test numbers 1 and 2 that are derived from test plan are shown in Tables 6 and 7, respectively. All the test cases have been tested successfully. Figures 10,11, 12, and 13 are the outputs of ultrasound medical images after being tampered in different ways. The result has shown that ROIDR has function as required, and its robustness to tampering has been tested successfully. The speed-up factor in Table 9 was generated by dividing the elapsed time of TALLOR and TALLOR-RS with ROI-DR (refer to Table 8), respectively. The average speed-up factor indicated in Table 9 has shown a significant improvement of time performance in ROI-DR watermarking scheme in relative to TALLOR and TALLOR-RS. The high average value in TALLOR-RS is due to the high values in non- tampering ROI; it is because TALLOR-RS will perform CRC checking from block to block in ROI until a tampering is detected. If no tampering has occurred, then CRC will be performed until the end of the blocks; therefore, non-tampered ROI consumes more time than the tampered ROI. The result in Table 10 has shown that if ROI has not been tampered with, then no recovery is necessary; therefore, no retrieval of stored ROI bits from RONI is required; thus, tampering on RONIs would be ignored. Tampering at left and right sides of RONI 3 gave a different result because ROI hash information is stored at the left side of RONI 3; therefore, only the tampering on the left side will affect the stored ROI hash values. The test data described in Table 10 are illustrated in Figs. 14, 15, and 16 . The watermarking algorithm is only able to detect the tampering occurrence within ROI area; thus, any tampering occurring beyond the ROI area would be ignored. The test data described in Table 11 are demonstrated in Fig. 17. The result in Table 12 indicated that RONIs will 


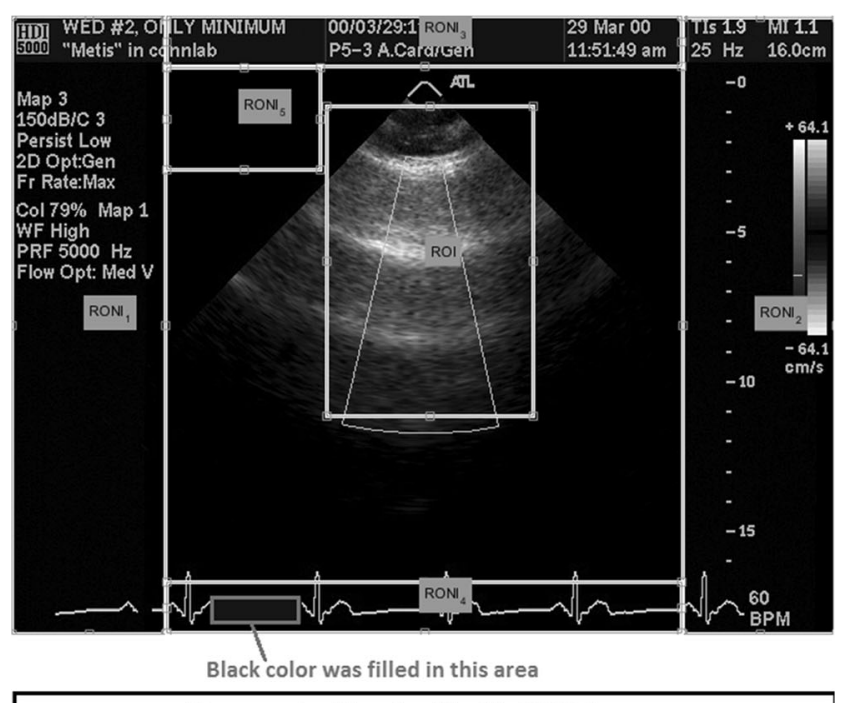

Tampered with color filled in RONI 4

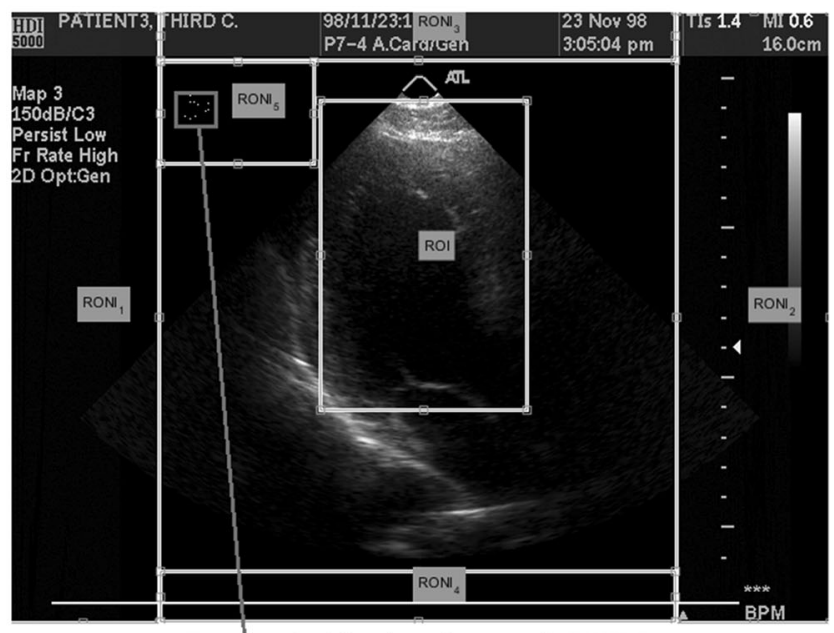

Tampered with salt and pepper in RONI 5

Fig. 16 Ultrasound medical images tampered on RONI 4 and RONI 5 be a concern if ROI has been tampered with. RONI 1 and RONI 2 are storing recovery bits; if either one of them is tampered with, then no recovery could be done. Tampering at ROI and RONI 4(B) caused an inequality in result comparison, which leads to a failure in ROI recovery. The example of tampering on both ROI and RONI has been demonstrated in Figs. 18, 19, 20, and 21.

\section{Conclusion}

The three important performance metrics as described previously have been successfully tested in ROI-DR watermarking scheme. Firstly, the generated watermarking is invisible in watermarked ultrasound medical image and has produced a satisfactory PSNR result. Secondly, the elapse time taken to perform watermarking embedding and authentication process has shown a high speed-up factor in relative to TALLOR and TALLOR-RS watermarking schemes. Thirdly, the robustness to tampering has been thoroughly tested by applying various kinds of tampering on ROI and RONI areas, and the result has shown that the detection and recovery function work as expected.

A comparison in algorithms, experiment, and result has been made among ROI-DR, TALLOR, and TALLOR-RS in Table 13. It has been shown that three of them have some similarity in algorithm, such as use of jpeg compression and Hash-256 and embedding two bits of each ROI pixel into two LSBs of each RONI pixel. They also have a same ROI size but with different RONI layout and organization. Both ROI-DR and TALLOR-RS are using segmentation technique, where ROI-DR watermarking scheme split ROI into two sections and stored them into
Fig. 17 Ultrasound medical images tampered at outside of ROI and RONI rectangles
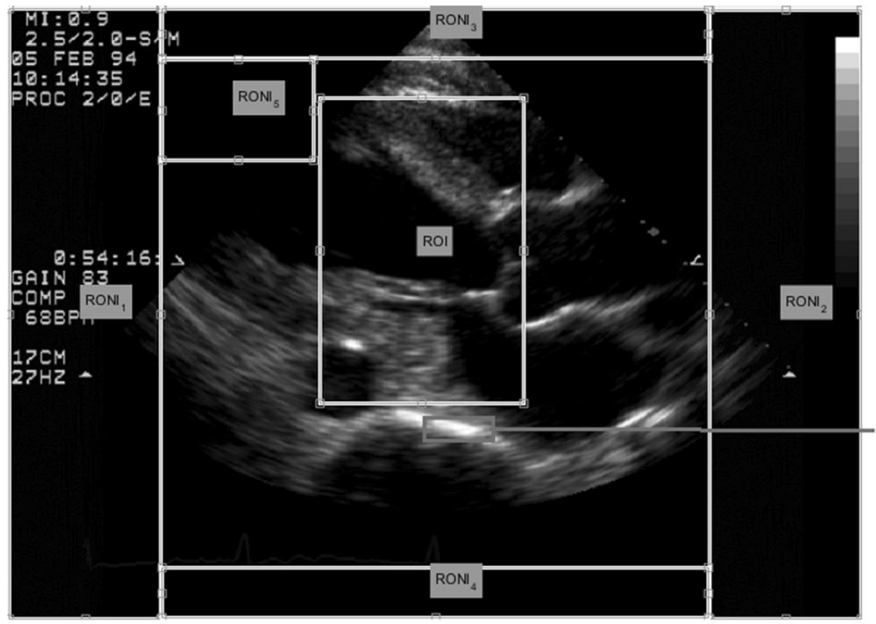

Tampered by cloning a portion of images

Tampered at outside of $\mathrm{ROI}$ and RONI rectangle 
Table 12 Test cases for test no. 6: test on where both ROI and RONI were tampered

\begin{tabular}{|c|c|c|c|c|}
\hline \multirow[t]{2}{*}{ Test data } & \multirow{2}{*}{$\begin{array}{l}\text { Elapsed } \\
\text { time }(\mathrm{s})\end{array}$} & \multicolumn{2}{|l|}{ Expected result } & \multirow[t]{2}{*}{ Actual result } \\
\hline & & Message display & $\begin{array}{l}\text { Produced } \\
\text { recovered file? }\end{array}$ & \\
\hline $\begin{array}{l}\text { Tampered at ROI and RONI } 1 \\
\text { (as shown in Fig. 18) }\end{array}$ & 0.446 & $\begin{array}{l}\text { ROI recovery failed because tampering has occurred on } \\
\text { RONI regions that contain ROI information!! }\end{array}$ & No & Same as expected result \\
\hline $\begin{array}{l}\text { Tampered at ROI and RONI } 2 \\
\text { (as shown in Fig. 18) }\end{array}$ & 0.410 & $\begin{array}{l}\text { ROI recovery failed because tampering has occurred on } \\
\text { RONI regions that contain ROI information!! }\end{array}$ & No & Same as expected result \\
\hline $\begin{array}{l}\text { Tampered at ROI and the right side of } \\
\text { RONI } 3 \text { (as shown in Fig. 19) }\end{array}$ & 0.595 & $\begin{array}{l}\text { Tampering has occurred on ROI!!! Recovery process } \\
\text { starts...file recovered successfully!! }\end{array}$ & Yes & Same as expected result \\
\hline $\begin{array}{l}\text { Tampered at ROI and the left side } \\
\text { of RONI } 3 \text { (as shown in Fig. 19) }\end{array}$ & 0.472 & $\begin{array}{l}\text { Tampering has occurred on ROI!!! Recovery process } \\
\text { starts...file recovered successfully!! }\end{array}$ & Yes & Same as expected result \\
\hline $\begin{array}{l}\text { Tampered at ROI and RONI } 4 \text { (A) } \\
\text { (as shown in Fig. 20) }\end{array}$ & 0.457 & $\begin{array}{l}\text { Tampering has occurred on ROI!!! Recovery process } \\
\text { starts... file recovered successfully!! }\end{array}$ & Yes & Same as expected result \\
\hline $\begin{array}{l}\text { Tampered at ROI and RONI } 4 \text { (B) } \\
\text { (as shown in Fig. 20) }\end{array}$ & 0.440 & $\begin{array}{l}\text { ROI recovery failed because tampering has occurred on } \\
\text { RONI regions that contain ROI information!! }\end{array}$ & No & Same as expected result \\
\hline $\begin{array}{l}\text { Tampered at ROI and RONI } 5 \\
\text { (as shown in Fig. 21) }\end{array}$ & 0.425 & $\begin{array}{l}\text { Tampering has occurred on ROI!!! Recovery process } \\
\text { starts... file recovered successfully!! }\end{array}$ & Yes & Same as expected result \\
\hline
\end{tabular}

Fig. 18 Ultrasound medical images tampered at ROI and RONI
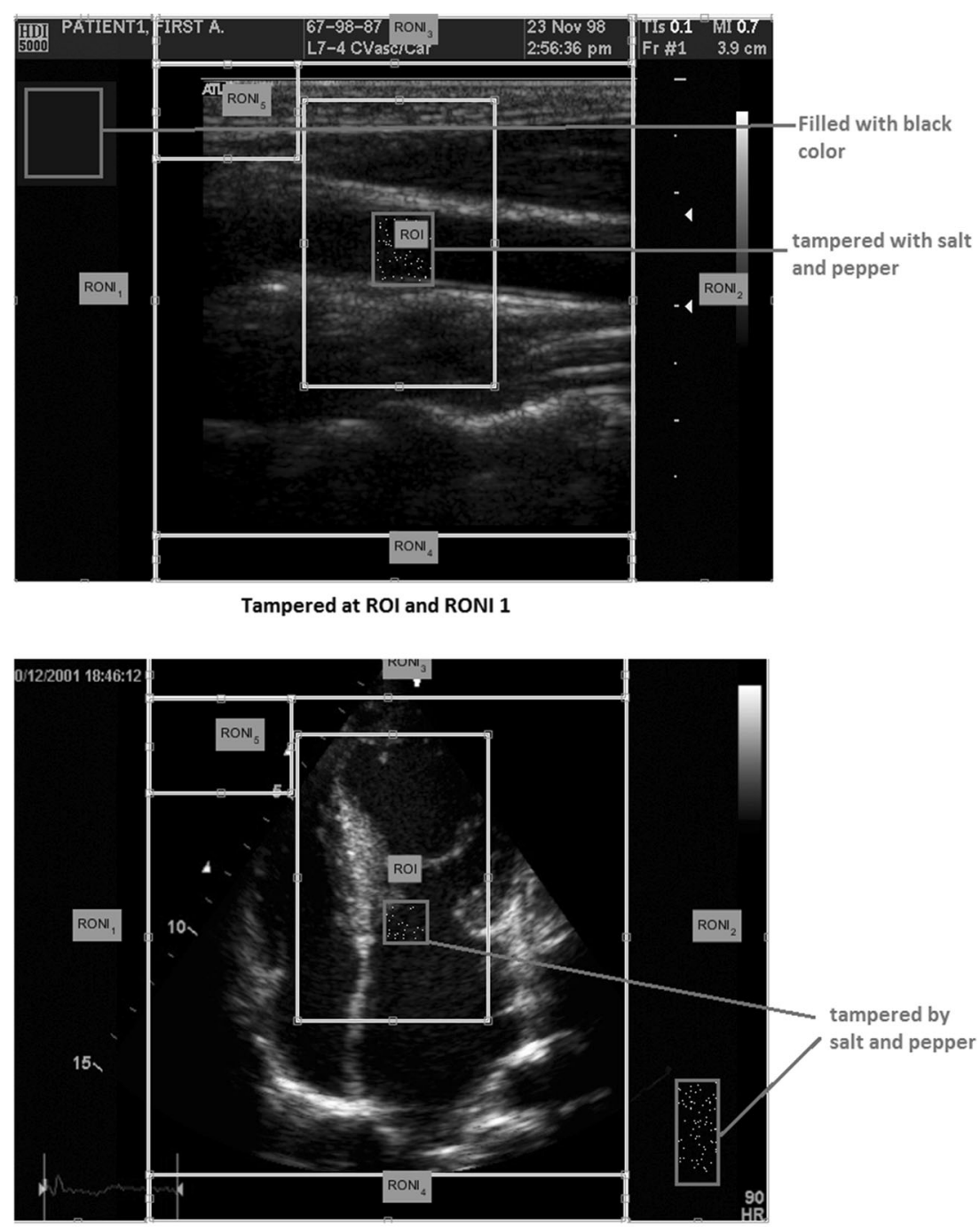

Tampered at ROI and RONI 2 
RONI 1 and RONI 2, respectively, whereas TALLOR-RS divided ROI into $40 \times 40$ blocks and implement each with CRC. Both TALLOR and TALLOR-RS are using block location and matrix concatenation method to store ROI bits before transfer to RONI LSB, which required high memory consumption, thus slowing down the process. Whereas ROI-DR watermarking scheme crops out the ROI and RONI image and pairs up 1 pixel ( 8 bits) of ROI to 4 pixels ( $4 \times 2$ bits) of RONI, then overwriting 2 bits of each ROI pixel into two LSBs of each RONI pixel iteratively is done. This process is more direct and has less memory consumption. Fairness of comparison of these three watermarking schemes was ensured by having them tested under the same environment and using the same set of ultrasound medical images as an input file for watermarking embedding process, and the watermarked ultrasound medical images are generated as a DICOM file. Same tampering technique was applied on watermarked ultrasound medical image for testing these three scheme authentication process. The result indicated that ROI-DR watermarking scheme has similar PSNR value $(\sim 48 \mathrm{~dB})$ and shorter elapsed time as compared to TALLOR and TALLOR-RS watermarking schemes. ROI-DR has average speed-up factors of 22.55 and 26.65 in relative to TALLOR and TALLOR-RS, respectively, in watermarking embedding process. Whereas, in watermarking authentication process, ROI-DR has average speed-up factors of 21.89 and 42.79 relative to TALLOR and TALLOR-RS, respectively. The high speed-up factor in relative to TALLOR-RS is due to that the time taken in authenticating non-tampered ultrasound medical images was too high, which compromised the average of speed-up factor in TALLOR-RS watermarking authentication process. This is because TALLOR-RS
Fig. 19 Ultrasound medical images tampered at ROI and RONI 3
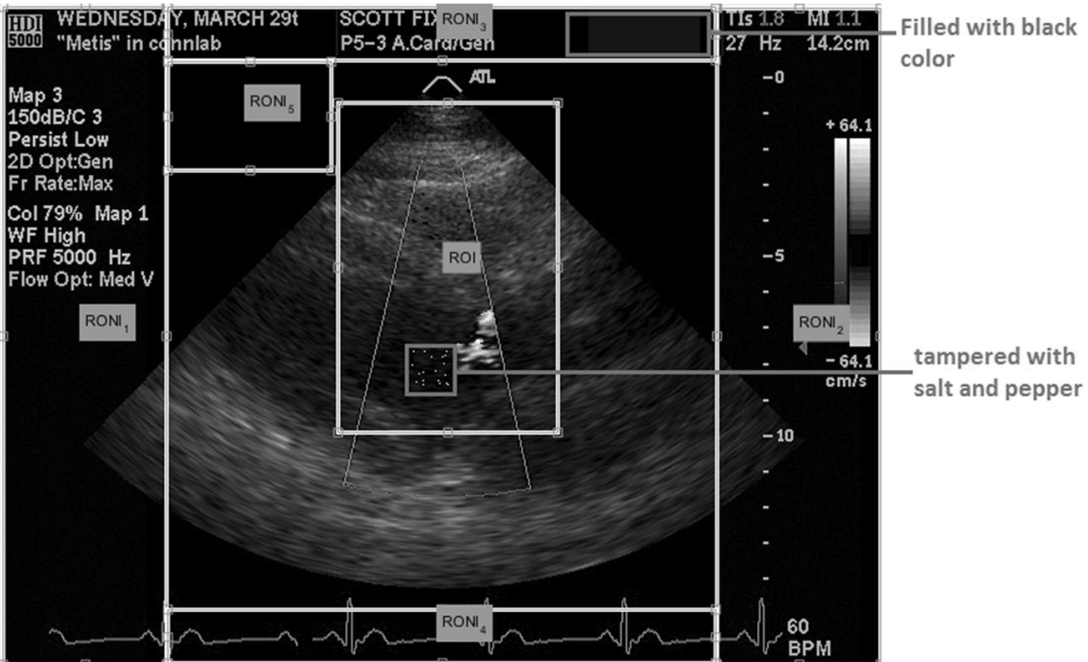

Tampered at ROI and right side of RONI 3
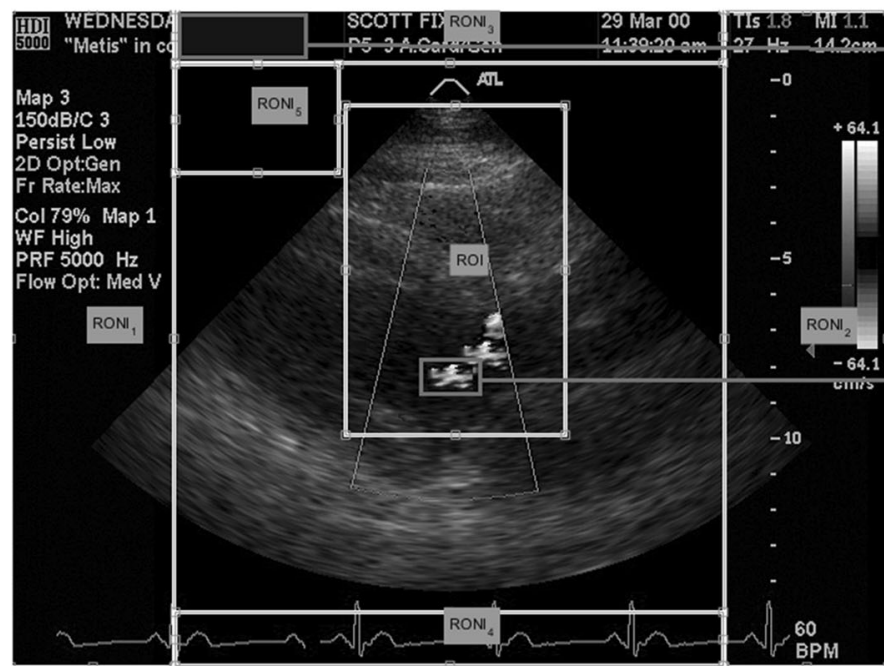

Filled with black color

Tampered at ROI and the left side of RONI 3 
Fig. 20 Ultrasound medical images tampered at ROI and RONI 4

Fig. 21 Ultrasound medical images tampered at ROI and RONI 5
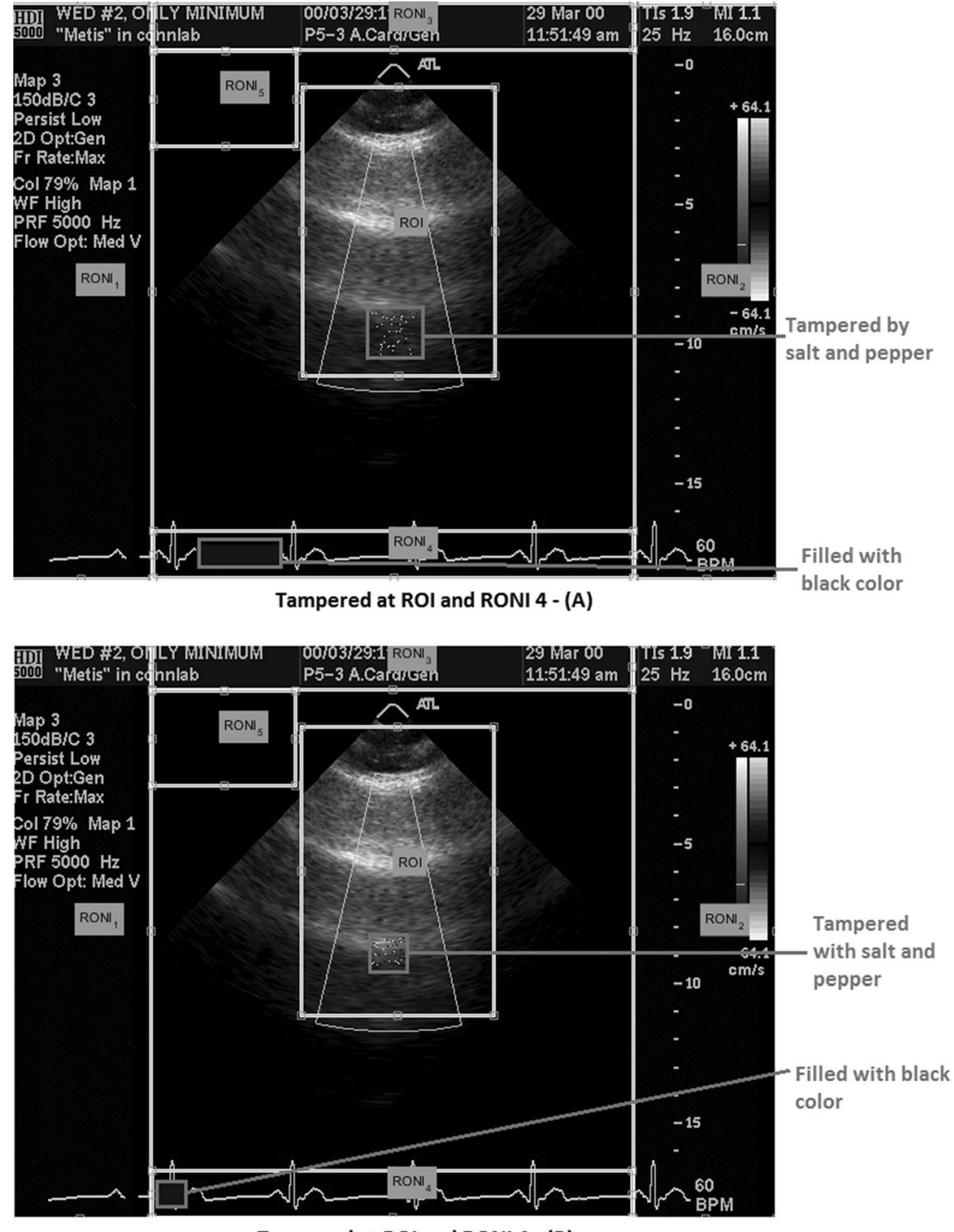

Tampered at ROI and RONI 4 - (B)

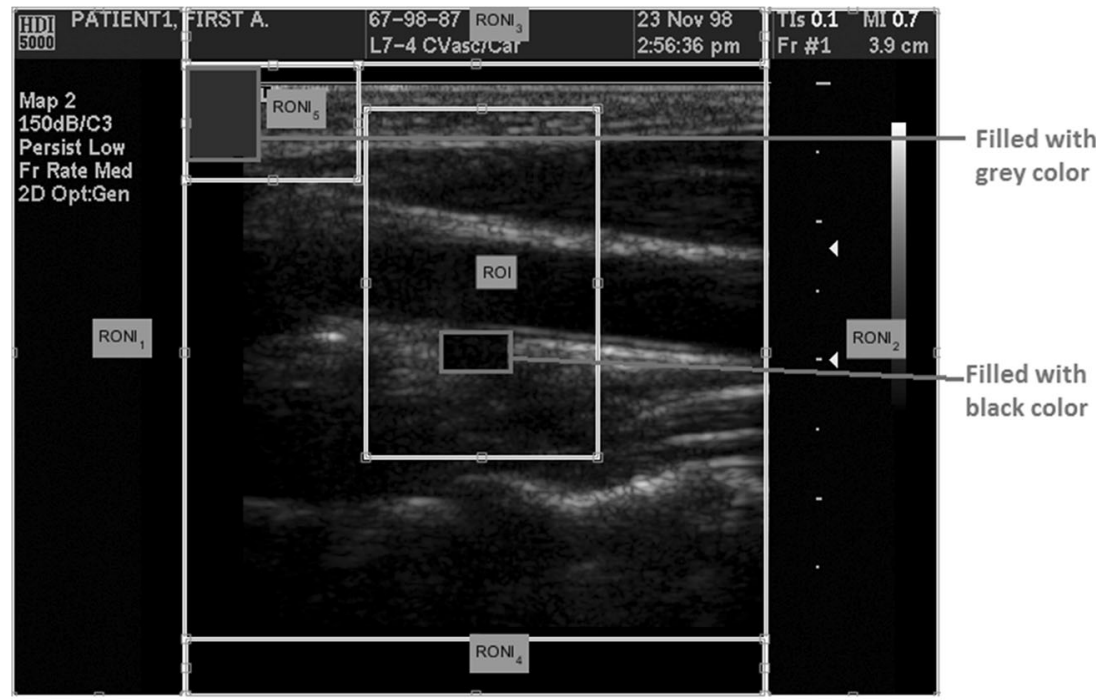

Tampered at ROI and RONI 5 
Table 13 A table of comparison between ROI-DR, TALLOR, and TALLOR-RS watermarking schemes

\begin{tabular}{|c|c|c|c|}
\hline \multirow[b]{2}{*}{ Watermarking scheme } & \multirow{2}{*}{$\begin{array}{l}\text { Current research } \\
\text { ROI-DR }\end{array}$} & \multicolumn{2}{|l|}{ Previous research [15] } \\
\hline & & TALLOR & TALLOR-RS \\
\hline \multicolumn{4}{|l|}{ Algorithm: } \\
\hline - ROI size & $160 \times 240$ pixels & $160 \times 240$ pixels & $160 \times 240$ pixels \\
\hline - Using Hash-256 & Yes & Yes & Yes \\
\hline - Using jpeg compression & Yes & Yes & Yes \\
\hline - Store ROI bits into 2 LSBs of RONI & Yes & Yes & Yes \\
\hline - Use segmentation & Split into 2 sections & No & $40 \times 40$ pixels \\
\hline - Use CRC & No & No & Yes \\
\hline $\begin{array}{l}\text { - Use matrix concatenation method in storing } \\
\text { ROI bits }\end{array}$ & No & Yes & Yes \\
\hline - Way of storing ROI bits into RONIs & $\begin{array}{l}\text { Split compressed ROI bits into } \\
\text { two and stored into RONI } 1 \text { and } \\
\text { RONI } 2\end{array}$ & $\begin{array}{l}\text { Store ROI bits into RONI circularly by } \\
\text { block location, wherein storing of the } \\
\text { coordinate } \\
\text { values of current stored bit location } \\
\text { takes place. }\end{array}$ & $\begin{array}{l}\text { Same as TALLOR } \\
\text { plus CRC }\end{array}$ \\
\hline - Way of image recovery & $\begin{array}{l}\text { Recovered by replacing the whole } \\
\text { tampered ROI with stored ROI } \\
\text { image. }\end{array}$ & $\begin{array}{l}\text { Recovered by replacing the tampered } \\
\text { pixels that have been localized. }\end{array}$ & $\begin{array}{l}\text { Recovered by } \\
\text { replacing the } \\
\text { tampered block. }\end{array}$ \\
\hline \multicolumn{4}{|l|}{ Experiment: } \\
\hline $\begin{array}{l}\text { - Use same set of ultrasound medical image } \\
\text { samples as input file for watermarking } \\
\text { embedding process }\end{array}$ & Yes & Yes & Yes \\
\hline $\begin{array}{l}\text { - Use same set of tampered images for } \\
\text { authentication process }\end{array}$ & Yes & Yes & Yes \\
\hline $\begin{array}{l}\text { - Conduct under the same hardware and } \\
\text { software environment. }\end{array}$ & Yes & Yes & Yes \\
\hline \multicolumn{4}{|l|}{ Result: } \\
\hline - Average of PSNR (dB) & 48.4141 & 48.413 & 48.943 \\
\hline $\begin{array}{l}\text { - Elapsed time in watermarking embedding } \\
\text { process }\end{array}$ & $0.98 \mathrm{~s}$ & $22.14 \mathrm{~s}$ & $26.09 \mathrm{~s}$ \\
\hline $\begin{array}{l}\text { - Elapsed time in watermarking authentication } \\
\text { process }\end{array}$ & $0.34 \mathrm{~s}$ & $7.10 \mathrm{~s}$ & $6.82 \mathrm{~s}$ \\
\hline - Able to detect tampering & Yes & Yes & Yes \\
\hline - Able to produce exact ROI recovery & Yes & Yes & Yes \\
\hline - Able to generate a recovered DICOM file & Yes & Yes & Yes \\
\hline
\end{tabular}

needs to go through the whole process of Cyclic Redundancy Check (CRC) checking even though no tampering has occurred. The actual average speed-up factor of ROI-DR in relative to TALLOR-RS could be counted as 16.36 if excluding the non-tampered ultrasound medical image in calculating the average of speed-up factor for watermarking authentication process. CRC checking in TALLOR-RS has sped up the authentication process in relative to TALLOR, but more time is required in implementing CRC into watermarking embedding process as compared to TALLOR. In conclusion, the current research, ROI-DR watermarking scheme, has maintained integrity of ultrasound medical images and improved the time performance significantly as compared to the previous research, TALLOR and TALLOR-RS watermarking schemes.

\section{References}

1. Guo X, Zhuang T: Lossless watermarking for verifying the integrity of medical images with tamper localization. J Digit Imaging 22: 620-628, 2008

2. Liew SC, Liew SW, Jasni MZ: Tamper localization and lossless recovery watermarking scheme with roi segmentation and multilevel authentication. J Digit Imaging 26(2):316-325, 2013

3. Rayachoti E, Edara SR: Robust medical image watermarking technique for accurate detection of tampers inside region of interest and recovering original region of interest. IET Image Process 9(8):615625,2015

4. Wenbo D, Chueh LP, Yong LG: An improved tamper detection and localization scheme for volumetric DICOM images. J Digit Imaging 25(6):751-763, 2012

5. Tan CK, Ng JC, Xu X, Poh CL, Guan YL, Sheah K: Security protection of dicom medical images using dual-layer reversible watermarking with tamper detection capability. J Digit Imaging 24(3):528-540, 2011 
6. Memon NA, Gilani SAM, Asad Ali: Watermarking of chest CT scan medical images for content authentication. Proceeding of 2009 International Conference on Information and Communication Technologies, IEEE, 2009, pp. 175-180

7. Fotopoulos V, Stavrinou ML, Skodras AN: Medical Image Authetication and Self Correction through an Adaptive Reversible Watermarking Technique. Proceedings of the 8th IEEE International Conference on BioInformatics and BioEngineering, IEEE, 2008, pp. 1-5. doi: 10.1109 /BIBE.2008.4696803

8. Huang H, Coatrieux G, Montagner J, Shu HZ, Luo LM, Roux Ch: Medical image integrity control seeking into the detail of the tampering. 2008 30th Annual International Conference of the IEEE Engineering in Medicine and Biology Society, IEEE, 2008, pp. 414-417. doi: 10.1109/IEMBS.2008.4649178

9. Jasni MZ and Abdul RF: Medical image watermarking with tamper detection and recovery. Proceedings of the 28th Annual International Conference of the IEEE Engineering in Medicine and Biology Society, IEEE, 2006, pp. 3270-3273

10. Al-Qershi OM, Khoo BE: Authentication and data hiding using a hybrid ROI-based watermarking scheme for DICOM images. J Digit Imaging 24(1):114-125, 2011

11. Badran EF, Sharkas MA and Attallah OA: Multiple watermark embedding scheme in wavelet-spatial domains based on ROI of medical images. In Radio Science Conference, 2009. NRSC 2009. National, IEEE, 2009, pp. 1-8

12. Coatrieux G, Puentes J, Roux C, Lamard M and Daccache W: January. A low distorsion and reversible watermark: application to angiographic images of the retina. In 2005 IEEE Engineering in Medicine and Biology 27th Annual Conference, IEEE, 2006, pp. 2224-2227

13. Shih FY, Wu YT: Robust watermarking and compression for medical images based on genetic algorithms. J Inf Sci 175(3):200-216, 2005

14. Lim SJ, Moon HM, Chae SH, Pan SB, Chung Y and Chang MH: December. Dual watermarking method for integrity of medical images. In 2008 Second International Conference on Future Generation Communication and Networking, Vol. 2, IEEE, 2008, pp. 70-73

15. Liew SC: Tamper Localization and recovery watermarking schemes for medical images in PACS. Ph.D. Thesis. Malayisa: Universiti Malayisa Pahang, 2011

16. Cox IJ, Miller ML, Bloom JA and Honsinger C: Digital watermarking (Vol. 1558607145). San Francisco: Morgan Kaufmann, 2002

17. Smitha B and Navas KA: Spatial domain-high capacity data hiding in ROI images. In 2007 International Conference on Signal Processing, Communications and Networking, IEEE, 2007, pp. $528-533$ 